\title{
The heat flow in an optimal Fréchet space of unbounded initial data in $\mathbb{R}^{d}$
}

\author{
James C. Robinson ${ }^{1}$, \\ Aníbal Rodríguez-Bernal ${ }^{2,}$ *†
}

March 22, 2019

\author{
${ }^{1}$ Mathematics Institute, Zeeman Building, \\ University of Warwick, \\ Coventry, CV4 7AL. U.K. \\ E-mail: j.c.robinson@warwick.ac.uk \\ ${ }^{2}$ Departamento de Matemática Aplicada \\ Universidad Complutense de Madrid \\ 28040 Madrid, Spain \\ and \\ Instituto de Ciencias Matemáticas \\ CSIC-UAM-UC3M-UCM ${ }^{3}$ \\ E-mail: arober@mat.ucm.es
}

\begin{abstract}
In this paper we show that solutions of the heat equation that are given in terms of the heat kernel define semigroups on the family of Fréchet spaces $L_{0}^{p}\left(\mathbb{R}^{d}\right)$, the intersection (over all $\varepsilon>0$ ) of the spaces $L_{\varepsilon}^{p}\left(\mathbb{R}^{d}\right)$ of functions such that $\int_{\mathbb{R}^{d}} \mathrm{e}^{-\varepsilon|x|^{2}}|f(x)|^{p} \mathrm{~d} x<\infty$. These spaces consist of functions that are 'large at infinity', and $L_{0}^{1}\left(\mathbb{R}^{d}\right)$ is the maximal space in which one can use the heat kernel to obtain globally-defined solutions of the heat equation. We prove suitable estimates from $L_{0}^{p}\left(\mathbb{R}^{d}\right)$ into $L_{0}^{q}\left(\mathbb{R}^{d}\right), q \geq p$, for these semigroups.

We then consider the heat semigroup posed in spaces that are dual to these spaces of functions, namely the spaces $L_{-\varepsilon}^{p}\left(\mathbb{R}^{d}\right)$ of very-rapidly decreasing functions such that $\int_{\mathbb{R}^{d}} \mathrm{e}^{\varepsilon|x|^{2}}|f(x)|^{p} \mathrm{~d} x<\infty$. We show that $\left(L_{p \varepsilon}^{p}\left(\mathbb{R}^{d}\right)\right)^{\prime}=L_{-q \varepsilon}^{q}\left(\mathbb{R}^{d}\right)$ (with $1<p<\infty$ and $(p, q)$ conjugate), and that the heat flow on $L_{\varepsilon}^{p}\left(\mathbb{R}^{d}\right)$ is the adjoint of the flow on $L_{-\delta}^{q}\left(\mathbb{R}^{d}\right)$ for an appropriate (time-dependent) choice of $\delta$.

\footnotetext{
*Partially supported by Project MTM2016-75465, MICINN and GR58/08 Grupo 920894, UCM, Spain

${ }^{\dagger}$ Partially supported by PRX17/00522 Programa Salvador de Madariaga MECyD, Spain and EPSRC grant $\mathrm{EP} / \mathrm{R} 023778 / 1$, UK.

${ }^{3}$ Partially supported by ICMAT Severo Ochoa project SEV-2015-0554 (MINECO)
} 


\section{Introduction}

In a previous paper, [6], we considered solutions of the heat equation

$$
u_{t}-\Delta u=0, x \in \mathbb{R}^{d}, t>0, \quad u(x, 0)=u_{0}(x) .
$$

with initial data in the spaces

$$
L_{\varepsilon}^{1}\left(\mathbb{R}^{d}\right):=\left\{f \in L_{\mathrm{loc}}^{1}\left(\mathbb{R}^{d}\right): \int_{\mathbb{R}^{d}} \mathrm{e}^{-\varepsilon|x|^{2}}|f(x)| \mathrm{d} x<\infty\right\},
$$

which are Banach spaces when equipped with the norm

$$
\|f\|_{L_{\varepsilon}^{1}\left(\mathbb{R}^{d}\right)}=\left(\frac{\varepsilon}{\pi}\right)^{d / 2} \int_{\mathbb{R}^{d}} \mathrm{e}^{-\varepsilon|x|^{2}}|f(x)| \mathrm{d} x .
$$

We showed that, despite the large growth of these functions at infinity, such solutions can be written using the heat kernel,

$$
u(x, t)=S(t) u_{0}(x):=\frac{1}{(4 \pi t)^{d / 2}} \int_{\mathbb{R}^{d}} \mathrm{e}^{-\frac{|x-y|^{2}}{4 t}} u_{0}(y) \mathrm{d} y, \quad x \in \mathbb{R}^{d}, t>0,
$$

and satisfy the estimate

$$
\|u(t)\|_{L_{\varepsilon(t)}^{1}\left(\mathbb{R}^{d}\right)} \leq\left\|u_{0}\right\|_{L_{\varepsilon}^{1}\left(\mathbb{R}^{d}\right)}, \quad \text { where } \quad \varepsilon(t):=\frac{\varepsilon}{1-4 \varepsilon t},
$$

for all $0 \leq t<1 / 4 \varepsilon$. Notice that in the estimates above, which will be shown to be optimal in Section 3 (see Proposition 3.4), the solution must be estimated in a norm that changes with time. In fact, as time advances, the estimate on the solution can only be performed in a progressively worse space. We showed in [6] that, since we allow initial data which may be very large at infinity, these solutions may not exist for all time, by a mechanism of mass moving from infinity; there we also characterised those initial data for which this happens and the points at which the solution blows up.

We also showed that if the initial condition $0 \leq u_{0} \in L_{\text {loc }}^{1}\left(\mathbb{R}^{d}\right)$ gives rise to a globally defined solution then we must have

$$
u_{0} \in L_{0}^{1}\left(\mathbb{R}^{d}\right):=\bigcap_{\varepsilon>0} L_{\varepsilon}^{1}\left(\mathbb{R}^{d}\right) .
$$

Conversely, for $u_{0} \in L_{0}^{1}\left(\mathbb{R}^{d}\right)$, (1.3) defines a global solution of the heat equation. Thus, the space (1.5) is an optimal class for the global existence of solutions given by (1.3).

The starting point for this paper is the observation that if $u_{0} \in L_{0}^{1}\left(\mathbb{R}^{d}\right)$ then for any $\delta>0$ and any $t>0$ one can rewrite the estimate in (1.4) as

$$
\left\|S(t) u_{0}\right\|_{L_{\delta}^{1}\left(\mathbb{R}^{d}\right)} \leq\left\|u_{0}\right\|_{L_{\delta(t)}^{1}\left(\mathbb{R}^{d}\right)}, \quad \text { where } \quad \delta(t):=\frac{\delta}{1+4 \delta t} ;
$$

this shows in particular that $S(t): L_{0}^{1}\left(\mathbb{R}^{d}\right) \rightarrow L_{0}^{1}\left(\mathbb{R}^{d}\right)$ for every $t>0$. Equipping $L_{0}^{1}\left(\mathbb{R}^{d}\right)$ with an appropriate topology (see Section 3) we thereby obtain a very natural example of a semigroup defined on a Fréchet space. 
It is possible to extend the rich classical theory for solutions of the heat equation in Lebesgue spaces to this context, in particular the $L^{p}-L^{q}$ estimates

$$
\|u(t)\|_{L^{q}\left(\mathbb{R}^{d}\right)} \leq(4 \pi t)^{-\frac{d}{2}\left(\frac{1}{p}-\frac{1}{q}\right)}\left\|u_{0}\right\|_{L^{p}\left(\mathbb{R}^{d}\right)}, \quad \text { for every } t>0 \text { and } 1 \leq p \leq q \leq \infty .
$$

Defining

$$
L_{\varepsilon}^{p}\left(\mathbb{R}^{d}\right):=\left\{f \in L_{\mathrm{loc}}^{p}\left(\mathbb{R}^{d}\right): \int_{\mathbb{R}^{d}} \mathrm{e}^{-\varepsilon|x|^{2}}|f(x)|^{p} \mathrm{~d} x<\infty\right\}
$$

with norm

$$
\|f\|_{L_{\varepsilon}^{p}\left(\mathbb{R}^{d}\right)}:=\left(\frac{\varepsilon}{\pi}\right)^{d / 2 p}\left(\int_{\mathbb{R}^{d}} \mathrm{e}^{-\varepsilon|x|^{2}}|f(x)|^{p} \mathrm{~d} x\right)^{1 / p},
$$

in Section 4 we obtain $L_{p \delta(t)}^{p}-L_{q \delta}^{q}$ estimates in line with (1.6), namely

$$
\|u(t)\|_{L_{q \delta}^{q}\left(\mathbb{R}^{d}\right)} \leq c_{p, q} \frac{(1+4 \delta t)^{\frac{d}{2}\left(1-\frac{1}{q}\right)}}{(4 \delta t)^{\frac{d}{2}\left(\frac{1}{p}-\frac{1}{q}\right)}}\left\|u_{0}\right\|_{L_{p \delta(t)}^{p}\left(\mathbb{R}^{d}\right)} .
$$

which hold for $u_{0} \in L_{0}^{p}\left(\mathbb{R}^{d}\right):=\bigcap_{\varepsilon>0} L_{\varepsilon}^{p}\left(\mathbb{R}^{d}\right)$. Taking $q=p$ shows that $S(t): L_{0}^{p}\left(\mathbb{R}^{d}\right) \rightarrow L_{0}^{p}\left(\mathbb{R}^{d}\right)$ for every $t>0$; we can use the solutions of the heat equation to define a semigroup on every member of this family of Fréchet spaces.

Notice that in (1.7) besides the typical term $t^{-\frac{d}{2}\left(\frac{1}{p}-\frac{1}{q}\right)}$ (see (1.6)) that describes the time singularity of the solution for short times, an algebraically increasing term $(1+4 \delta t)^{\frac{d}{2}\left(1-\frac{1}{q}\right)}$ appears, reflecting the fact that we are dealing with functions that are very large at infinity. This term reflects the mechanism of mass moving from infinity that was shown in [6] to be able to produce nonlinear-like behaviour in the solutions of (1.1).

The estimate in (1.7) shows another feature that reflects the fact that our chosen space of initial conditions allows for large growth at infinity: to estimate the solution at time $t$ in a fixed space $L_{q \delta}^{q}\left(\mathbb{R}^{d}\right)$, it is necessary to measure the initial data in the space $L_{p \delta(t)}^{p}\left(\mathbb{R}^{d}\right)$ that changes with time, requiring better and better integrability of $u_{0}$ as $t$ increases, since $\delta(t)$ is decreasing.

In our previous paper [6] we used some duality arguments, pairing solutions of the equation for initial data in $L_{0}^{1}\left(\mathbb{R}^{d}\right)$ with solutions corresponding to initial data with compact support. In the second half of this paper we present a much more systematic duality theory.

We define spaces of 'very rapidly decaying' functions, by setting

$$
L_{-\varepsilon}^{p}\left(\mathbb{R}^{d}\right):=\left\{f \in L_{\mathrm{loc}}^{p}\left(\mathbb{R}^{d}\right): \int_{\mathbb{R}^{d}} \mathrm{e}^{\varepsilon|x|^{2}}|f(x)|^{p} \mathrm{~d} x<\infty\right\} \quad \text { for } 1 \leq p<\infty,
$$

with the norm

$$
\|f\|_{L_{-\varepsilon}^{p}\left(\mathbb{R}^{d}\right)}:=\left(\frac{\pi}{\varepsilon}\right)^{d / 2 p}\left(\int_{\mathbb{R}^{d}} \mathrm{e}^{\varepsilon|x|^{2}}|f(x)|^{p} \mathrm{~d} x\right)^{\frac{1}{p}} .
$$

We show in Proposition 6.4 that for $1<p<\infty$ the dual of $L_{p \varepsilon}^{p}\left(\mathbb{R}^{d}\right)$ is $L_{-p^{\prime} \varepsilon}^{p^{\prime}}\left(\mathbb{R}^{d}\right)$, where $p$ and $p^{\prime}$ are conjugate (and corresponding results for the endpoint values of $p$ ). Defining

$$
L_{-0}^{p}\left(\mathbb{R}^{d}\right):=\cup_{\varepsilon>0} L_{-\varepsilon}^{p}\left(\mathbb{R}^{d}\right)
$$


we deduce that $\left(L_{0}^{p}\left(\mathbb{R}^{d}\right)\right)^{\prime}=L_{-0}^{p^{\prime}}\left(\mathbb{R}^{d}\right)$ in Corollary 6.5.

In Corollary 6.7 we obtain $L^{p}-L^{q}$ type estimates for the action of the heat semigroup in these spaces of very-rapidly decaying functions. In the final section we show that the heat flow in the $L_{\varepsilon}^{p}\left(\mathbb{R}^{d}\right)$ spaces is the adjoint of the heat flow in the $L_{-\varepsilon}^{p}\left(\mathbb{R}^{d}\right)$ spaces in the sense that for each $t>0$

$$
\int_{\mathbb{R}^{d}}\left[S(t) u_{0}\right] \varphi=\int_{\mathbb{R}^{d}} u_{0}[S(t) \varphi]
$$

for $u_{0} \in L_{p \delta(t)}^{p}\left(\mathbb{R}^{d}\right)$ and $\varphi \in L_{-p^{\prime} \delta}^{p^{\prime}}\left(\mathbb{R}^{d}\right)$, where $\delta(t):=\delta /(1+4 \delta t)$.

The results from the first half of the paper form an important ingredient in our analysis of the non-autonomous problem

$$
u_{t}-\Delta u+\lambda u=f(t)
$$

and the associated elliptic equation

$$
-\Delta u+\lambda u=f
$$

in a forthcoming paper [7].

\section{Some previous results}

In this section we quote some results from [6] that will be needed below. We consider the Cauchy problem

$$
u_{t}-\Delta u=0, x \in \mathbb{R}^{d}, t>0, \quad u(x, 0)=u_{0}(x),
$$

whose solutions are expected to be given in terms of the heat kernel by

$$
u\left(x, t ; u_{0}\right)=S(t) u_{0}(x):=\frac{1}{(4 \pi t)^{d / 2}} \int_{\mathbb{R}^{d}} \mathrm{e}^{-\frac{|x-y|^{2}}{4 t}} u_{0}(y) \mathrm{d} y,
$$

if $u_{0} \in L_{\mathrm{loc}}^{1}\left(\mathbb{R}^{d}\right)$ or, more generally, if $u_{0}$ is a Radon measure $u_{0} \in \mathcal{M}_{\mathrm{loc}}\left(\mathbb{R}^{d}\right)$ by

$$
u\left(x, t ; u_{0}\right)=S(t) u_{0}(x):=\frac{1}{(4 \pi t)^{d / 2}} \int_{\mathbb{R}^{d}} \mathrm{e}^{-\frac{|x-y|^{2}}{4 t}} \mathrm{~d} u_{0}(y)
$$

Notice that the space of Radon measures in $\mathbb{R}^{d}$ is defined as $\mathcal{M}_{\text {loc }}\left(\mathbb{R}^{d}\right)=\left(C_{c}\left(\mathbb{R}^{d}\right)\right)^{\prime}$ where in $C_{c}\left(\mathbb{R}^{d}\right)$ we consider the final topology for the inclusions $C_{c}(K) \hookrightarrow C_{c}\left(\mathbb{R}^{d}\right), K \subset \subset \mathbb{R}^{d}$, see [2,3].

In [6], solutions of the form (2.2) for equation (2.1) were constructed for initial data in the very large spaces of measures

$$
\mathcal{M}_{\varepsilon}\left(\mathbb{R}^{d}\right):=\left\{\mu \in \mathcal{M}_{\mathrm{loc}}\left(\mathbb{R}^{d}\right): \int_{\mathbb{R}^{d}} \mathrm{e}^{-\varepsilon|x|^{2}} \mathrm{~d}|\mu(x)|<\infty\right\} ;
$$

i.e. $\mathrm{e}^{-\varepsilon|x|^{2}} \in L^{1}(d|\mu|)$, for $\varepsilon>0$, where $|\mu|$ denotes the total variation of $\mu$. It is natural to endow this space with the norm

$$
\|\mu\|_{\mathcal{M}_{\varepsilon}\left(\mathbb{R}^{d}\right)}:=\left(\frac{\varepsilon}{\pi}\right)^{d / 2} \int_{\mathbb{R}^{d}} \mathrm{e}^{-\varepsilon|x|^{2}} \mathrm{~d}|\mu(x)|
$$


with which it becomes a Banach space. This space contains in particular the class of point functions

$$
L_{\varepsilon}^{1}\left(\mathbb{R}^{d}\right):=\left\{f \in L_{\mathrm{loc}}^{1}\left(\mathbb{R}^{d}\right): \int_{\mathbb{R}^{d}} \mathrm{e}^{-\varepsilon|x|^{2}}|f(x)| \mathrm{d} x<\infty\right\} ;
$$

with the norm

$$
\|f\|_{L_{\varepsilon}^{1}\left(\mathbb{R}^{d}\right)}:=\left(\frac{\varepsilon}{\pi}\right)^{d / 2} \int_{\mathbb{R}^{d}} \mathrm{e}^{-\varepsilon|x|^{2}}|f(x)| \mathrm{d} x
$$

for which a positive constant function $f(x) \equiv c$ has norm $c$. This space is also a Banach space. If $f \in L_{\varepsilon}^{1}\left(\mathbb{R}^{d}\right)$ then $f \in \mathcal{M}_{\varepsilon}\left(\mathbb{R}^{d}\right)$ and $\|f\|_{\mathcal{M}_{\varepsilon}\left(\mathbb{R}^{d}\right)}=\|f\|_{L_{\varepsilon}^{1}\left(\mathbb{R}^{d}\right)}$.

We consider the family of integrable weights

$$
\rho_{\varepsilon}(x)=\left(\frac{\varepsilon}{\pi}\right)^{d / 2} \mathrm{e}^{-\varepsilon|x|^{2}}, \quad \int_{\mathbb{R}^{d}} \rho_{\varepsilon}(x) \mathrm{d} x=1,
$$

with $\varepsilon>0$ and note that if $0<\varepsilon_{1}<\varepsilon_{2}$ then

$$
\rho_{\varepsilon_{1}}(x) \geq\left(\frac{\varepsilon_{1}}{\varepsilon_{2}}\right)^{d / 2} \rho_{\varepsilon_{2}}(x) ; \quad x \in \mathbb{R}^{d}
$$

hence for $\mu \in \mathcal{M}_{\varepsilon_{1}}\left(\mathbb{R}^{d}\right)$

$$
\|\mu\|_{\mathcal{M}_{\varepsilon_{2}}\left(\mathbb{R}^{d}\right)} \leq\left(\frac{\varepsilon_{2}}{\varepsilon_{1}}\right)^{d / 2}\|\mu\|_{\mathcal{M}_{\varepsilon_{1}}\left(\mathbb{R}^{d}\right)}
$$

and therefore $\mathcal{M}_{\varepsilon}\left(\mathbb{R}^{d}\right)$ and $L_{\varepsilon}^{1}\left(\mathbb{R}^{d}\right)$ are increasing in $\varepsilon>0$ and

The following result is taken from Theorem 3.6 in [6].

Theorem 2.1. Suppose that $u_{0} \in \mathcal{M}_{\varepsilon}\left(\mathbb{R}^{d}\right)$. Set $T(\varepsilon)=1 / 4 \varepsilon$, and let $u(x, t)$ be given by $(2.2)$.

Then $u(t) \in L_{\mathrm{loc}}^{\infty}\left(\mathbb{R}^{d}\right)$ for $t \in(0, T(\varepsilon)), u \in C^{\infty}\left(\mathbb{R}^{d} \times(0, T(\varepsilon))\right)$, and u satisfies (2.1) pointwise in $\mathbb{R}^{d} \times(0, T(\varepsilon))$. Furthermore for every $\varphi \in C_{c}\left(\mathbb{R}^{d}\right)$ and $0 \leq t<T(\varepsilon)$

$$
\int_{\mathbb{R}^{d}} \varphi u(t)=\int_{\mathbb{R}^{d}} S(t) \varphi \mathrm{d} u_{0}
$$

and

$$
\int_{\mathbb{R}^{d}} \varphi u(t) \rightarrow \int_{\mathbb{R}^{d}} \varphi \mathrm{d} u_{0}
$$

i.e. $u(t) \rightarrow u_{0}$ as $t \rightarrow 0^{+}$in the sense of measures.

It is also clear that if $0 \leq u_{0} \in \mathcal{M}_{\varepsilon}\left(\mathbb{R}^{d}\right)$ is non-zero then $u(x, t)>0$ for all $x \in \mathbb{R}^{d}$, $t \in(0, T(\varepsilon))$, i.e. the Strong Maximum Principle holds.

Conversely it was proved in Lemma 3.2 in [6] that if $0 \leq u_{0} \in \mathcal{M}_{\mathrm{loc}}\left(\mathbb{R}^{d}\right), u(x, t)$ is given by (2.2) and satisfies $u(x, t)<\infty$ for some $x \in \mathbb{R}^{d}, t>0$ then

$$
u_{0} \in \mathcal{M}_{\varepsilon}\left(\mathbb{R}^{d}\right) \text { for every } \varepsilon>1 / 4 t .
$$

Hence the spaces (2.3) are the natural ones in which to consider solutions of (2.1) that can be written in the form $(2.2)$.

Some additional properties of the solutions in Theorem 2.1 are given in the following result, see Proposition 3.7 in [6]. 
Proposition 2.2. Suppose that $u_{0} \in \mathcal{M}_{\varepsilon}\left(\mathbb{R}^{d}\right)$, set $T(\varepsilon)=1 / 4 \varepsilon$, and let $u(x, t)$ be given by $(2.2)$. Then

(i) For $0<t<T(\varepsilon)$ and for any $\delta \geq \frac{1}{4(T(\varepsilon)-t)}>\varepsilon$ we have $u(t) \in L_{\delta}^{1}\left(\mathbb{R}^{d}\right)$. Moreover if we set

$$
\varepsilon(t):=\frac{1}{4(T(\varepsilon)-t)}=\frac{\varepsilon}{(1-4 \varepsilon t)}
$$

then

$$
\|u(t)\|_{L_{\varepsilon(t)}^{1}\left(\mathbb{R}^{d}\right)} \leq\left\|u_{0}\right\|_{\mathcal{M}_{\varepsilon}\left(\mathbb{R}^{d}\right)}
$$

(ii) For $0 \leq s<t<T(\varepsilon)$

$$
u(t)=S(t-s) u(s) .
$$

(iii) For any multi-index $\alpha \in \mathbb{N}^{d}$, for $0<t<T(\varepsilon)$ and for any $\delta>\frac{1}{4(T(\varepsilon)-t)}>\varepsilon$ we have $D_{x}^{\alpha} u(t) \in L_{\delta}^{1}\left(\mathbb{R}^{d}\right)$. Moreover for any $\gamma>1$ and $0<t<\frac{T(\varepsilon)}{\gamma}$ and if we set

$$
\delta(t):=\frac{1}{4(T(\varepsilon)-\gamma t)}=\frac{\varepsilon}{(1-4 \varepsilon \gamma t)}
$$

then

$$
\left\|D_{x}^{\alpha} u(t)\right\|_{L_{\delta(t)}^{1}\left(\mathbb{R}^{d}\right)} \leq \frac{c_{\alpha, \gamma}}{t^{\frac{|\alpha|}{2}}}\left\|u_{0}\right\|_{\mathcal{M}_{\varepsilon}\left(\mathbb{R}^{d}\right)} .
$$

(iv) For any multi-index $\alpha \in \mathbb{N}^{d}, m \in \mathbb{N}$ and for each $t_{0} \in(0, T(\varepsilon))$ there exists $\delta\left(t_{0}\right)>\varepsilon$ such that the mapping $(0, T(\varepsilon)) \ni t \mapsto D_{x, t}^{\alpha, m} u(t)$ is continuous in $L_{\delta\left(t_{0}\right)}^{1}\left(\mathbb{R}^{d}\right)$ at $t=t_{0}$.

Remark 2.3. (i) Notice that for any multi-index $\alpha \in \mathbb{N}^{d}$ and any $0<\beta<1$ (with even $\beta=0$ if $\alpha=0$ from (2.2))

$$
\left|D_{x}^{\alpha} u(x, t)\right| \leq \frac{c_{\alpha, \beta}}{t^{d / 2+|\alpha| / 2}} \int_{\mathbb{R}^{d}} \mathrm{e}^{-(1-\beta) \frac{|x-y|^{2}}{4 t}} \mathrm{~d}\left|u_{0}(y)\right| .
$$

From here we can get that for any $0<\gamma<1$,

$$
\left|D_{x}^{\alpha} u(x, t)\right| \leq \frac{c_{\alpha, \beta}}{t^{d / 2+|\alpha| / 2}} \mathrm{e}^{\left(\frac{1}{\gamma}-1\right)(1-\beta) \frac{|x|^{2}}{4 t}} \int_{\mathbb{R}^{d}} \mathrm{e}^{-(1-\gamma)(1-\beta) \frac{|y|^{2}}{4 t}} \mathrm{~d}\left|u_{0}(y)\right| .
$$

Estimates (2.11) and (2.12) are at the core of the proof that $u \in C^{\infty}\left(\mathbb{R}^{d} \times(0, T(\varepsilon))\right)$ in Theorem 2.1 and of parts (iii) and (iv) of Proposition 2.2; they will be used again below.

(ii) The index $\delta\left(t_{0}\right)$ in part (iv) of Proposition 2.2 can be taken as $\delta\left(t_{0}\right)=(1-\gamma)(1-\beta) \frac{1}{4 t_{0}}>\varepsilon$, provided that $t_{0}<T:=(1-\gamma)(1-\beta) T(\varepsilon)$.

Notice that (2.9) implies that the solution lies, at each time, in a space that progressively deteriorates, since $\varepsilon(t)$ is increasing. That is, the integrability of the solution worsens with time. This estimate will be improved to an equality for non-negative initial data later in Proposition 3.4 , showing in particular that it is optimal.

The solutions above attain the initial data in the following sense, see Corollaries 3.8 and 3.9 in $[6]$. 
Corollary 2.4. If $u_{0} \in \mathcal{M}_{\varepsilon}\left(\mathbb{R}^{d}\right)$, let $u(x, t)$ be given by (2.2) and $\varphi \in C_{0}\left(\mathbb{R}^{d}\right)$ is such that $|\varphi(x)| \leq A \mathrm{e}^{-\gamma|x|^{2}}, x \in \mathbb{R}^{d}$, with $\gamma>\varepsilon$. Then

$$
\int_{\mathbb{R}^{d}} u(t) \varphi \rightarrow \int_{\mathbb{R}^{d}} \varphi \mathrm{d} u_{0} \quad \text { as } \quad t \rightarrow 0 .
$$

If $u_{0} \in L_{\varepsilon}^{1}\left(\mathbb{R}^{d}\right)$ then for any $\delta>\varepsilon$ we have

$$
u(t) \rightarrow u_{0} \quad \text { in } \quad L_{\delta}^{1}\left(\mathbb{R}^{d}\right) \quad \text { as } t \rightarrow 0^{+} .
$$

Additionally (i) if $u_{0} \in L_{\mathrm{loc}}^{p}\left(\mathbb{R}^{d}\right)$ with $1 \leq p<\infty$ then $u(t) \rightarrow u_{0}$ in $L_{\mathrm{loc}}^{p}\left(\mathbb{R}^{d}\right)$ as $t \rightarrow 0^{+}$; (ii) if $u_{0} \in C\left(\mathbb{R}^{d}\right)$ then $u(t) \rightarrow u_{0}$ in $L_{\mathrm{loc}}^{\infty}\left(\mathbb{R}^{d}\right)$ as $t \rightarrow 0^{+}$.

Finally, a uniqueness result was obtained in Theorem 4.1 in [6].

Observe that for $u_{0} \in \mathcal{M}_{\varepsilon}\left(\mathbb{R}^{d}\right)$ the solution of the heat equation in Theorem 2.1 satisfies

$$
u\left(0, t ;\left|u_{0}\right|\right)=\frac{1}{(4 \pi t)^{d / 2}} \int_{\mathbb{R}^{d}} \mathrm{e}^{-\frac{|y|^{2}}{4 t}} \mathrm{~d}\left|u_{0}(y)\right|
$$

and so

$$
\left\|u_{0}\right\|_{\mathcal{M}_{\varepsilon}\left(\mathbb{R}^{d}\right)}=u\left(0, \frac{1}{4 \varepsilon},\left|u_{0}\right|\right)=S\left(\frac{1}{4 \varepsilon}\right)\left|u_{0}\right|(0) .
$$

Therefore estimates (2.9) and (2.10) imply that we can estimate the solution of the heat equation by its value at $x=0$.

\section{The heat semigroup in the Fréchet spaces $L_{0}^{1}\left(\mathbb{R}^{d}\right)$ and $\mathcal{M}_{0}\left(\mathbb{R}^{d}\right)$}

As a consequence of the above results above we define

$$
L_{0}^{1}\left(\mathbb{R}^{d}\right)=\bigcap_{\varepsilon>0} L_{\varepsilon}^{1}\left(\mathbb{R}^{d}\right) \subset \mathcal{M}_{0}\left(\mathbb{R}^{d}\right)=\bigcap_{\varepsilon>0} \mathcal{M}_{\varepsilon}\left(\mathbb{R}^{d}\right) .
$$

Proposition 3.1. If $u_{0} \in \mathcal{M}_{0}\left(\mathbb{R}^{d}\right)$ then $u(x, t)$, given by $(2.2)$, is well defined for all $x \in \mathbb{R}^{d}$ and $t>0$.

Conversely, if $0 \leq u_{0} \in \mathcal{M}_{\mathrm{loc}}\left(\mathbb{R}^{d}\right)$ and $u(x, t)$ is defined for all $t>0$ then $u_{0} \in \mathcal{M}_{0}\left(\mathbb{R}^{d}\right)$.

This shows that the spaces (3.1) are optimal for globally-defined solutions of (2.1) that are given by (2.2). Our goal in this paper is to analyze the heat flow given by (2.2) in these spaces.

Observe that in the spaces

$$
L_{0}^{1}\left(\mathbb{R}^{d}\right)=\bigcap_{\varepsilon>0} L_{\varepsilon}^{1}\left(\mathbb{R}^{d}\right) \quad \text { and } \quad \mathcal{M}_{0}\left(\mathbb{R}^{d}\right)=\bigcap_{\varepsilon>0} \mathcal{M}_{\varepsilon}\left(\mathbb{R}^{d}\right)
$$

the expressions in (2.5) and (2.4), respectively, define a family of seminorms. It follows that $L_{0}^{1}\left(\mathbb{R}^{d}\right)$ and $\mathcal{M}_{0}\left(\mathbb{R}^{d}\right)$ are Fréchet spaces ${ }^{4}$.

\footnotetext{
${ }^{4}$ Recall that a Fréchet space is a vector space $X$ equipped with a countable family $\left\{p_{j}\right\}_{j=1}^{\infty}$ of seminorms with respect to which $X$ is complete: if $\left(x_{n}\right)_{n=1}^{\infty}$ is a sequence in $X$ that is Cauchy with respect to every seminorm $p_{j}$ then there exists $x \in X$ such that $p_{j}\left(x_{n}-x\right) \rightarrow 0$ for every $j$.
} 
Lemma 3.2. The spaces $L_{0}^{1}\left(\mathbb{R}^{d}\right)$ and $\mathcal{M}_{0}\left(\mathbb{R}^{d}\right)$ with the family of norms (2.5) and (2.4) respectively, are Fréchet spaces, where a sequence $\left(u_{n}\right)$ converges to $u_{0}$ in $\mathcal{M}_{0}\left(\mathbb{R}^{d}\right)$ (respectively in $L_{0}^{1}\left(\mathbb{R}^{d}\right)$ ) if and only if $u_{n} \rightarrow u_{0}$ in $\mathcal{M}_{\varepsilon}\left(\mathbb{R}^{d}\right)$ (resp. in $L_{\varepsilon}^{1}\left(\mathbb{R}^{d}\right)$ ) for every $\varepsilon>0$. Convergence in $\mathcal{M}_{0}\left(\mathbb{R}^{d}\right)$ (resp. in $L_{0}^{1}\left(\mathbb{R}^{d}\right)$ ) implies convergence in $\mathcal{M}_{\mathrm{loc}}\left(\mathbb{R}^{d}\right)$ (resp. in $L_{\mathrm{loc}}^{1}\left(\mathbb{R}^{d}\right)$ ).

Proof. We prove the result in $L_{0}^{1}\left(\mathbb{R}^{d}\right)$. The case of $\mathcal{M}_{0}\left(\mathbb{R}^{d}\right)$ follows similar lines. Just note that from (2.8) it is enough to consider a countable family of the norms (2.5) corresponding to a decreasing sequence $\varepsilon_{k} \rightarrow 0$. This defines a locally convex topology in $L_{0}^{1}\left(\mathbb{R}^{d}\right)$. Since each space $L_{\varepsilon}^{1}\left(\mathbb{R}^{d}\right)$ is a Banach space it follows that $L_{0}^{1}\left(\mathbb{R}^{d}\right)$ is complete, and hence a Fréchet space. The rest is immediate.

Now we prove that the heat equation (1.1) defines a semigroup of solution operators in the Fréchet spaces $L_{0}^{1}\left(\mathbb{R}^{d}\right)$ and $\mathcal{M}_{0}\left(\mathbb{R}^{d}\right)$.

Proposition 3.3. The family of mappings defined by

$$
u(t)=S(t) u_{0}, \quad u_{0} \in \mathcal{M}_{0}\left(\mathbb{R}^{d}\right), \quad t \geq 0
$$

as in $(2.2)$, satisfy $S(t): \mathcal{M}_{0}\left(\mathbb{R}^{d}\right) \longrightarrow L_{0}^{1}\left(\mathbb{R}^{d}\right)$, are linear, continuous and order preserving. Furthermore, $\{S(t)\}_{t \geq 0}$ is a continuous semigroup in $L_{0}^{1}\left(\mathbb{R}^{d}\right)$ and in $\mathcal{M}_{0}\left(\mathbb{R}^{d}\right)$. In the former space the semigroup is continuous at $t=0$. In the latter, the semigroup is continuous at $t=0$ in the sense of measures.

Finally, for any $\delta>0$ we have

$$
\left\|S(t) u_{0}\right\|_{L_{\delta}^{1}\left(\mathbb{R}^{d}\right)} \leq\left\|u_{0}\right\|_{\mathcal{M}_{\delta(t)}\left(\mathbb{R}^{d}\right)}, \quad t>0
$$

where

$$
\delta(t):=\frac{\delta}{1+4 \delta t} .
$$

The inequality in (3.2) becomes an equality if $u_{0} \geq 0$.

Also for any $\delta>0, \gamma>1$ and any multi-index $\alpha \in \mathbb{N}^{d}$ we have

$$
\left\|D_{x}^{\alpha} S(t) u_{0}\right\|_{L_{\delta}^{1}\left(\mathbb{R}^{d}\right)} \leq \frac{c_{\alpha, \gamma}}{t^{\frac{|\alpha|}{2}}}\left\|u_{0}\right\|_{\mathcal{M}_{\tilde{\delta}(t)}\left(\mathbb{R}^{d}\right)}, \quad t>0,
$$

with $\tilde{\delta}(t)=\frac{\delta}{1+4 \gamma \delta t}$. In particular, for $t>0, D_{x}^{\alpha} S(t): \mathcal{M}_{0}\left(\mathbb{R}^{d}\right) \longrightarrow L_{0}^{1}\left(\mathbb{R}^{d}\right)$ is linear and continuous.

Proof. From part (i) in Proposition 2.2 it follows that $S(t): \mathcal{M}_{0}\left(\mathbb{R}^{d}\right) \longrightarrow L_{0}^{1}\left(\mathbb{R}^{d}\right)$ is well defined for every $t>0$. Indeed for any fixed $t>0$ and $u_{0} \in \mathcal{M}_{0}\left(\mathbb{R}^{d}\right)$, take any $\delta>0$ and choose $\varepsilon>0$ such that $\delta>\varepsilon, 0<t<T(\varepsilon)=1 / 4 \varepsilon$ and $\delta=\frac{1}{4(T(\varepsilon)-t)}$, that is $\varepsilon=\delta(t)=\frac{\delta}{1+4 \delta t}$. Then (2.9) gives (3.2). This in turn gives $S(t) u_{0} \in L_{0}^{1}\left(\mathbb{R}^{d}\right)$ and the continuity of $S(t): \mathcal{M}_{0}\left(\mathbb{R}^{d}\right) \longrightarrow L_{0}^{1}\left(\mathbb{R}^{d}\right)$. Now part (ii) in Proposition 2.2 implies that $\{S(t)\}_{t \geq 0}$ is a semigroup in both $L_{0}^{1}\left(\mathbb{R}^{d}\right)$ and $\mathcal{M}_{0}\left(\mathbb{R}^{d}\right)$. The order-preserving property follows from Theorem 2.1. We will show that the inequality in (3.2) becomes an equality if $u_{0} \geq 0$ as part of Proposition 3.4 below. 
Now we prove that the solution curves of the semigroup are continuous in $L_{0}^{1}\left(\mathbb{R}^{d}\right)$. To see this, fix any $u_{0} \in \mathcal{M}_{0}\left(\mathbb{R}^{d}\right)$ and $t_{0}>0$. Using part (iv) of Proposition 2.2 with $\alpha=0$ and part (ii) in Remark 2.3, given $\delta>0$, chose $\beta=0,0<\gamma<1$ and $\varepsilon>0$ such that $\delta>(1-\gamma) \frac{1}{4 t_{0}}>\varepsilon$, that is, $t_{0}<T:=(1-\gamma) T(\varepsilon)$. Then $t \mapsto u(t)$ is continuous in $L_{\delta}^{1}\left(\mathbb{R}^{d}\right)$ at $t=t_{0}$.

Also, if $u_{0} \in L_{0}^{1}\left(\mathbb{R}^{d}\right)$ then Corollary 2.4 implies the semigroup is continuous at $t=0$ in $L_{\delta}^{1}\left(\mathbb{R}^{d}\right)$ for any $\delta>0$. On the other hand, Theorem 2.1 implies that the semigroup is continuous at $t=0$ in $\mathcal{M}_{0}\left(\mathbb{R}^{d}\right)$ in the sense of measures.

Now if for any $t>0$ and $\gamma>1$ we chose $\tilde{\varepsilon}>0$ such that and $0<t<\frac{T(\tilde{\varepsilon})}{\gamma}$ and

$$
\delta=\frac{1}{4(T(\tilde{\varepsilon})-\gamma t)}=\frac{\tilde{\varepsilon}}{(1-4 \tilde{\varepsilon} \gamma t)},
$$

that is $\tilde{\varepsilon}=\tilde{\delta}(t)=\frac{\delta}{1+4 \gamma \delta t}$ then $(2.10)$ gives $(3.3)$.

Before continuing, and as mentioned above, we will first improve (2.9) in Proposition 2.2 [and hence (3.2) in Proposition 3.3] to equalities for non-negative initial data.

Proposition 3.4. Assume that $0 \leq u_{0} \in \mathcal{M}_{\varepsilon}\left(\mathbb{R}^{d}\right)$ and let $u(x, t)$ be given by (2.2).

(i) For every $\delta>\varepsilon$ and $0 \leq t \leq \frac{1}{4 \varepsilon}-\frac{1}{4 \delta}=T(\varepsilon)-T(\delta)$

$$
\|u(t)\|_{L_{\delta}^{1}\left(\mathbb{R}^{d}\right)}=\left\|u_{0}\right\|_{\mathcal{M}_{\delta(t)}\left(\mathbb{R}^{d}\right)}
$$

with $\delta(t)=\frac{\delta}{1+4 \delta t}$. In particular, this estimate holds for any $\delta>0$ and $t>0$ whenever $0 \leq u_{0} \in \mathcal{M}_{0}\left(\mathbb{R}^{d}\right)$.

(ii) For $0 \leq t<T(\varepsilon)=\frac{1}{4 \varepsilon}$ and for $\varepsilon(t):=\frac{1}{4(T(\varepsilon)-t)}=\frac{\varepsilon}{(1-4 \varepsilon t)}$ we have

$$
\|u(t)\|_{L_{\varepsilon(t)}^{1}\left(\mathbb{R}^{d}\right)}=\left\|u_{0}\right\|_{\mathcal{M}_{\varepsilon}\left(\mathbb{R}^{d}\right)} .
$$

Proof. Since $u_{0} \geq 0$ for $\delta>0$ we have

$$
\int_{\mathbb{R}^{d}} \mathrm{e}^{-\delta|x|^{2}} u(x, t) \mathrm{d} x=\frac{1}{(4 \pi t)^{d / 2}} \int_{\mathbb{R}^{d}} \int_{\mathbb{R}^{d}} \mathrm{e}^{-\delta|x|^{2}} \mathrm{e}^{-|x-z|^{2} / 4 t} \mathrm{~d} u_{0}(z) \mathrm{d} x .
$$

Notice that completing the square we obtain

$$
\delta|x|^{2}+\frac{|x-z|^{2}}{4 t}=\frac{1+4 \delta t}{4 t}\left|x-\frac{1}{1+4 \delta t} z\right|^{2}+\frac{\delta}{1+4 \delta t}|z|^{2}
$$

and so

$$
\int_{\mathbb{R}^{d}} \mathrm{e}^{-\delta|x|^{2}} u(x, t) \mathrm{d} x=\frac{1}{(4 \pi t)^{d / 2}} \int_{\mathbb{R}^{d}} \mathrm{e}^{-\frac{\delta}{1+4 \delta t}|z|^{2}} \mathrm{~d} u_{0}(z) \int_{\mathbb{R}^{d}} \mathrm{e}^{-\frac{1+4 \delta t}{4 t}\left|x-\frac{1}{1+4 \delta t} z\right|^{2}} \mathrm{~d} x .
$$

Since

$$
\int_{\mathbb{R}^{d}} \mathrm{e}^{-\frac{1+4 \delta t}{4 t}\left|x-\frac{1}{1+4 \delta t} z\right|^{2}} \mathrm{~d} x=\int_{\mathbb{R}^{d}} \mathrm{e}^{-\frac{1+4 \delta t}{4 t}|x|^{2}} \mathrm{~d} x=\left(\frac{4 \pi t}{1+4 \delta t}\right)^{d / 2}
$$


it follows that

$$
\int_{\mathbb{R}^{d}} \mathrm{e}^{-\delta|x|^{2}} u(x, t) \mathrm{d} x=\left(\frac{1}{1+4 \delta t}\right)^{d / 2} \int_{\mathbb{R}^{d}} \mathrm{e}^{-\frac{\delta}{1+4 \delta t}|z|^{2}} \mathrm{~d} u_{0}(z),
$$

that is

$$
\|u(t)\|_{L_{\delta}^{1}\left(\mathbb{R}^{d}\right)}=\left\|u_{0}\right\|_{\mathcal{M}_{\delta(t)}\left(\mathbb{R}^{d}\right)}
$$

with $\delta(t)=\frac{\delta}{1+4 \delta t}$.

First, since $\delta(t)$ is decreasing, for $\delta>\varepsilon$ the right hand side is defined up to the time for which $\delta(t)=\varepsilon$, that is, for $0 \leq t \leq \frac{1}{4 \varepsilon}-\frac{1}{4 \delta}=T(\varepsilon)-T(\delta)$. that is

Second, given $t$ with $0<t<T(\varepsilon)$, choose $\delta=\varepsilon(t)=\frac{1}{4(T(\varepsilon)-t)}=\frac{\varepsilon}{(1-4 \varepsilon t)}$ and then $\delta(t)=\varepsilon$,

$$
\|u(t)\|_{L_{\varepsilon(t)}^{1}\left(\mathbb{R}^{d}\right)}=\left\|u_{0}\right\|_{\mathcal{M}_{\varepsilon}\left(\mathbb{R}^{d}\right)} .
$$

Now we prove that the semigroup curves are more regular in space and time.

Proposition 3.5. For each $u_{0} \in \mathcal{M}_{0}\left(\mathbb{R}^{d}\right)$ and any multi-index $\alpha \in \mathbb{N}^{d}$, the solution curve

$$
(0, \infty) \ni t \longmapsto D_{x}^{\alpha} S(t) u_{0} \in L_{0}^{1}\left(\mathbb{R}^{d}\right)
$$

is $C^{\infty}$. If additionally,

$$
\sup _{\varepsilon>0}\left\|u_{0}\right\|_{\mathcal{M}_{\varepsilon}\left(\mathbb{R}^{d}\right)}<\infty
$$

then the solution curve is $C^{\omega}$, i.e. analytic, in $(0, \infty)$.

Proof. For any multi-index $\alpha \in \mathbb{N}^{d}$, for any $u_{0} \in \mathcal{M}_{0}\left(\mathbb{R}^{d}\right), t_{0}>0$ and $\delta>0$, part (iii) in Proposition 2.2 implies $D_{x}^{\alpha} u\left(t_{0}\right) \in L_{\delta}^{1}\left(\mathbb{R}^{d}\right)$ provided we chose $\delta>\frac{1}{4\left(T(\varepsilon)-t_{0)}\right)}>\varepsilon$, i.e. $\varepsilon<\frac{\delta}{1+4 \delta t_{0}}$. Hence $D_{x}^{\alpha} u\left(t_{0}\right) \in L_{0}^{1}\left(\mathbb{R}^{d}\right)$. Also using part (iv) of Proposition 2.2 and part (ii) in Remark 2.3, given $\delta>0$, chose $0<\beta, \gamma<1$ and $\varepsilon>0$ such that $\delta>(1-\beta)(1-\gamma) \frac{1}{4 t_{0}}>\varepsilon$, that is, $t_{0}<T:=(1-\beta)(1-\gamma) T(\varepsilon)$. Then $t \mapsto D_{x}^{\alpha} u(t)$ is continuous in $L_{\delta}^{1}\left(\mathbb{R}^{d}\right)$ at $t=t_{0}$.

Now we prove the time regularity of the solution curves. For this we first prove that they are differentiable in $L_{0}^{1}\left(\mathbb{R}^{d}\right)$ with $\frac{\mathrm{d}}{\mathrm{d} t} S(t) u_{0}=\partial_{t} S(t) u_{0}=\Delta S(t) u_{0}$. For this, for $t>0$ and $\delta>0$, we show that

$$
I(h)=\frac{S(t+h) u_{0}-S(t) u_{0}}{h}-\partial_{t} S(t) u_{0}
$$

goes to zero in $L_{\delta}^{1}\left(\mathbb{R}^{N}\right)$, as $h \rightarrow 0$. For this, note that

$$
I(h)(x)=\int_{\mathbb{R}^{d}}\left(\frac{K(x-y, t+h)-K(x-y, t)}{h}-\partial_{t} K(x-y, t)\right) \mathrm{d} u_{0}(y)
$$

where $K(x, t)=(4 \pi t)^{-d / 2} \mathrm{e}^{-\frac{|x|^{2}}{4 t}}$ denotes the heat kernel. Note then that we have

$$
\begin{aligned}
I(h)(x) & =\int_{\mathbb{R}^{d}}\left(\partial_{t} K(x-y, t+\theta h)-\partial_{t} K(x-y, t)\right) \mathrm{d} u_{0}(y) \\
& =\int_{\mathbb{R}^{d}} \theta h \partial_{t}^{2} K(x-y, t+\tilde{\theta} h) \mathrm{d} u_{0}(y) \\
& =h \int_{\mathbb{R}^{d}} \theta \Delta^{2} K(x-y, t+\tilde{\theta} h) \mathrm{d} u_{0}(y)
\end{aligned}
$$


for some $\theta, \tilde{\theta} \in[0,1]$ depending on $x, y$. Now we take $a>0$ small enough and $|h| \leq a t$. Now the kernel $H(x-y, t)=\theta \Delta^{2} K(x-y, t+\tilde{\theta} h)$ can be bounded in modulus by a term of the form $c \mathrm{e}^{-(1-\beta) \frac{|x-y|^{2}}{4 t}}$ with $0<\beta<1$ small enough. Now for any $\delta>0$ and $t>0$, choose $\varepsilon>0$ such that $0<t<T(\varepsilon)$ and $\delta>(1-\gamma)(1-\beta) \frac{1}{4 t}>\varepsilon$ for some $0<\gamma<1$. Now as in (2.11) and (2.12) we obtain that the integral term in the expression above for $I(h)$ is bounded in $L_{\delta}^{1}\left(\mathbb{R}^{d}\right)$. Hence $I(h) \rightarrow 0$ in that space as $h \rightarrow 0$.

In a completely analogous way we obtain

$$
\frac{\mathrm{d}^{m}}{\mathrm{~d} t^{m}} S(t) u_{0}=\partial_{t}^{m} S(t) u_{0}=(-\Delta)^{m} S(t) u_{0}
$$

which we have already proved are continuous in time in $L_{\delta}^{1}\left(\mathbb{R}^{d}\right)$ for any $\delta>0$. Therefore the curves of the semigroup are $C^{\infty}\left((0, \infty), L_{0}^{1}\left(\mathbb{R}^{N}\right)\right)$.

With the same arguments

$$
\frac{\mathrm{d}^{m}}{\mathrm{~d} t^{m}} D_{x}^{\alpha} S(t) u_{0}=\partial_{t}^{m} D_{x}^{\alpha} S(t) u_{0}=(-\Delta)^{m} D_{x}^{\alpha} S(t) u_{0}
$$

and are continuous in time in $L_{\delta}^{1}\left(\mathbb{R}^{d}\right)$ for any $\delta>0$ and hence in $L_{0}^{1}\left(\mathbb{R}^{d}\right)$.

Finally we prove the analyticity of the curves of the semigroup. For this we will prove that for each fixed $t>0$ and any $\delta>0$ the Taylor series

$$
\sum_{m=0}^{\infty} \frac{(z-t)^{m}}{m !} \frac{\mathrm{d}^{m}}{\mathrm{~d} t^{m}} S(t) u_{0}
$$

converges in $L_{\delta}^{1}\left(\mathbb{R}^{d}\right)$. For this we prove that for any $t>0$ and $m \in \mathbb{N}$

$$
\frac{\mathrm{d}^{m}}{\mathrm{~d} t^{m}} S(t) u_{0}=\partial_{t}^{m} S(t) u_{0}=(-\Delta)^{m} S(t) u_{0}=\left((-\Delta) S\left(\frac{t}{m}\right)\right)^{m} u_{0}
$$

and in this case from (3.3) we obtain

$$
\left\|\frac{\mathrm{d}^{m}}{\mathrm{~d} t^{m}} S(t) u_{0}\right\|_{L_{\delta}^{1}\left(\mathbb{R}^{d}\right)} \leq \frac{\left(c_{2} m\right)^{m}}{t^{m}}\left\|u_{0}\right\|_{\mathcal{M}_{\varepsilon_{m}}\left(\mathbb{R}^{d}\right)}
$$

with $\varepsilon_{j}$ for $j=1, \ldots, m-1$ satisfy $\varepsilon_{j+1}<g\left(\varepsilon_{j}\right)=\frac{\varepsilon_{j}}{1+4 \varepsilon_{j} \frac{t}{m}}, \varepsilon_{0}=\delta$. Hence if (3.4) is satisfied the series converges in $L_{\delta}^{1}\left(\mathbb{R}^{N}\right)$.

To prove (3.5) for $m=2$ we observe that for $t>0$ and $h$ small

$$
\begin{aligned}
\frac{\mathrm{d}^{2}}{\mathrm{~d} t^{2}} S(t) u_{0} & =\lim _{h \rightarrow 0} \frac{(-\Delta)\left(S(t+h) u_{0}-S(t) u_{0}\right)}{h} \\
& =\lim _{h \rightarrow 0}(-\Delta) S(t / 2) \frac{\left(S(t / 2+h) u_{0}-S(t / 2) u_{0}\right)}{h} .
\end{aligned}
$$

From the previous step we know that

$$
\lim _{h \rightarrow 0} \frac{\left(S(t / 2+h) u_{0}-S(t / 2) u_{0}\right)}{h}=(-\Delta) S(t / 2) u_{0}
$$


in $L_{0}^{1}\left(\mathbb{R}^{d}\right)$ and since $(3.3)$ implies $D_{x}^{\alpha} S(t): L_{0}^{1}\left(\mathbb{R}^{d}\right) \rightarrow L_{0}^{1}\left(\mathbb{R}^{d}\right)$ is continuous, it follows that

$$
\frac{\mathrm{d}^{2}}{\mathrm{~d} t^{2}} S(t) u_{0}=(-\Delta) S(t / 2)(-\Delta) S(t / 2) u_{0}
$$

in $L_{0}^{1}\left(\mathbb{R}^{d}\right)$.

Now, assuming that (3.5) is true for $m$ we have

$$
\begin{aligned}
\frac{\mathrm{d}^{m+1}}{\mathrm{~d} t^{m+1}} S(t) u_{0} & =\lim _{h \rightarrow 0}(-\Delta)^{m} \frac{\left(S(t+h) u_{0}-S(t) u_{0}\right)}{h} \\
& =\lim _{h \rightarrow 0}(-\Delta)^{m} S\left(\frac{m}{m+1} t\right) \frac{\left(S\left(\frac{t}{m+1}+h\right) u_{0}-S\left(\frac{t}{m+1}\right) u_{0}\right)}{h} .
\end{aligned}
$$

Again

$$
\lim _{h \rightarrow 0} \frac{S\left(\frac{t}{m+1}+h\right) u_{0}-S\left(\frac{t}{m+1}\right) u_{0}}{h}=(-\Delta) S\left(\frac{t}{m+1}\right) u_{0}
$$

in $L_{0}^{1}\left(\mathbb{R}^{d}\right)$ and the continuity of $(-\Delta)^{m} S\left(\frac{m}{m+1} t\right): L_{0}^{1}\left(\mathbb{R}^{d}\right) \rightarrow L_{0}^{1}\left(\mathbb{R}^{d}\right)$ leads to

$$
\frac{\mathrm{d}^{m+1}}{\mathrm{~d} t^{m+1}} S(t) u_{0}=(-\Delta)^{m} S\left(\frac{m}{m+1} t\right)(-\Delta) S\left(\frac{t}{m+1}\right) u_{0} ;
$$

now use that

$$
(-\Delta)^{m} S\left(\frac{m}{m+1} t\right)=\left((-\Delta) S\left(\frac{t}{m+1}\right)\right)^{m}
$$

to get

$$
\frac{\mathrm{d}^{m+1}}{\mathrm{~d} t^{m+1}} S(t) u_{0}=\left((-\Delta) S\left(\frac{t}{m+1}\right)\right)^{m+1} u_{0}
$$

To justify the last step above observe that in general we have

$$
S(s)(-\Delta) S(t)=-\Delta S(t+s) \quad t, s>0 .
$$

To see this note that

$$
-\Delta S(t+s) u_{0}=\lim _{h \rightarrow 0} \frac{S(t+s+h) u_{0}-S(t+s) u_{0}}{h}=\lim _{h \rightarrow 0} S(s) \frac{S(t+h) u_{0}-S(t) u_{0}}{h}
$$

and again we use $\lim _{h \rightarrow 0} \frac{S(t+h) u_{0}-S(t) u_{0}}{h}=(-\Delta) S(t) u_{0}$ in $L_{0}^{1}\left(\mathbb{R}^{d}\right)$ and the continuity of $S(s)$ in that space.

The proof of (3.4) leads us naturally to define the following spaces of measures or functions.

Definition 3.6. The space $\mathcal{M}_{0, B}\left(\mathbb{R}^{d}\right)$ is the subspace of $\mathcal{M}_{0}\left(\mathbb{R}^{d}\right)$ consisting of measures such that

$$
\left\|u_{0}\right\|_{\mathcal{M}_{0, B}\left(\mathbb{R}^{d}\right)}:=\sup _{\varepsilon>0}\left\|u_{0}\right\|_{\mathcal{M}_{\varepsilon}\left(\mathbb{R}^{d}\right)}<\infty .
$$

Analogously, $L_{0, B}^{1}\left(\mathbb{R}^{d}\right)$ is the subspace of $L_{0}^{1}\left(\mathbb{R}^{d}\right)$ consisting of functions such that

$$
\left\|u_{0} \mid\right\|_{L_{0, B}^{1}\left(\mathbb{R}^{d}\right)}:=\sup _{\varepsilon>0}\left\|u_{0}\right\|_{L_{\varepsilon}^{1}\left(\mathbb{R}^{d}\right)}<\infty ;
$$

clearly $\left\|u_{0}\right\|\left\|_{\mathcal{M}_{0, B}\left(\mathbb{R}^{d}\right)}=\right\|\left\|u_{0}\right\|_{L_{0, B}^{1}\left(\mathbb{R}^{d}\right)}$ for $u_{0} \in L_{0, B}^{1}\left(\mathbb{R}^{d}\right)$. 
Notice that these are very large spaces containing all the Lebesgue spaces $L^{p}\left(\mathbb{R}^{d}\right)$ and all the uniform Lebesgue spaces $L_{U}^{p}\left(\mathbb{R}^{N}\right)$ for $1 \leq p \leq \infty$. Also $\mathcal{M}_{0, B}\left(\mathbb{R}^{d}\right)$ contains the space of uniform measures $\mathcal{M}_{U}\left(\mathbb{R}^{d}\right)$; see $[6]$.

Before we discuss the action of the semigroup $S(t)$ on these spaces, we first prove some of their properties.

Lemma 3.7. The spaces $L_{0, B}^{1}\left(\mathbb{R}^{d}\right) \subset \mathcal{M}_{0, B}\left(\mathbb{R}^{d}\right)$ are proper subsets of $L_{0}^{1}\left(\mathbb{R}^{d}\right)$ and $\mathcal{M}_{0}\left(\mathbb{R}^{d}\right)$, respectively, and with the norms (3.9) and (3.8) are Banach spaces such that

$$
L_{U}^{1}\left(\mathbb{R}^{d}\right) \subset L_{0, B}^{1}\left(\mathbb{R}^{d}\right) \subset L_{0}^{1}\left(\mathbb{R}^{d}\right) \quad \text { and } \quad \mathcal{M}_{U}\left(\mathbb{R}^{d}\right) \subset \mathcal{M}_{0, B}\left(\mathbb{R}^{d}\right) \subset \mathcal{M}_{0}\left(\mathbb{R}^{d}\right)
$$

with continuous inclusions.

Furthermore, $\mathcal{M}_{0, B}\left(\mathbb{R}^{d}\right)$ can be characterized as the space of all $u_{0} \in \mathcal{M}_{\mathrm{loc}}\left(\mathbb{R}^{d}\right)$ such that the solution of the heat equation (1.1) given by $(2.2)$ is defined for all $t>0$ and $u\left(\cdot, \cdot ; u_{0}\right)$ is uniformly bounded in sets $\frac{|x|}{\sqrt{t}} \leq R$, with $R>0$.

Proof. Notice that it is immediate that (3.9) is a norm and $L_{0, B}^{1}\left(\mathbb{R}^{d}\right) \subset L_{0}^{1}\left(\mathbb{R}^{d}\right)$ with continuous inclusion. If $\left\{u_{n}\right\}_{n} \subset L_{0, B}^{1}\left(\mathbb{R}^{d}\right)$ is a Cauchy sequence in the norm (3.9) then clearly $u_{n} \rightarrow u_{0}$ in $L_{0}^{1}\left(\mathbb{R}^{d}\right)$ for some $u_{0} \in L_{0}^{1}\left(\mathbb{R}^{d}\right)$ and also $\left\|u_{n}\right\|_{L_{0, B}^{1}\left(\mathbb{R}^{d}\right)} \leq C$ independent of $n \in \mathbb{N}$. Hence $u_{0} \in L_{0, B}^{1}\left(\mathbb{R}^{d}\right)$. Also for any $\varepsilon>0$, some $n_{0} \in \mathbb{N}$ and all $n, m \geq n_{0}$ we have, for any $\delta>0$,

$$
\left\|u_{n}-u_{m}\right\|_{L_{\delta}^{1}\left(\mathbb{R}^{d}\right)} \leq \varepsilon .
$$

Taking the limit as $m \rightarrow \infty$ we get $u_{n} \rightarrow u_{0}$ in $L_{0, B}^{1}\left(\mathbb{R}^{d}\right)$ and this space is complete.

The proof for $\mathcal{M}_{0, B}\left(\mathbb{R}^{d}\right)$ follows the same lines.

The characterization of $\mathcal{M}_{0, B}\left(\mathbb{R}^{d}\right)$ follows from Proposition 6.1 in [6]. Also that $\mathcal{M}_{0, B}\left(\mathbb{R}^{d}\right)$ is a proper subspace of $\mathcal{M}_{0}\left(\mathbb{R}^{d}\right)$ follows from Section 6.2 in [6]. Finally, that $\mathcal{M}_{U}\left(\mathbb{R}^{d}\right) \subset \mathcal{M}_{0, B}\left(\mathbb{R}^{d}\right)$ and $L_{U}^{1}\left(\mathbb{R}^{d}\right) \subset L_{0, B}^{1}\left(\mathbb{R}^{d}\right)$ follows from Proposition 5.2 in [6] and the characterization above.

The following result concerning the solutions of the heat equation with initial data in $L_{0, B}^{1}\left(\mathbb{R}^{d}\right)$ or $\mathcal{M}_{0, B}\left(\mathbb{R}^{d}\right)$ is essentially immediate from Propositions 3.3 and 3.5 , since the constants in the estimates (3.2), (3.3), and (3.6) do not depend on $\delta$.

Proposition 3.8. The spaces $L_{0, B}^{1}\left(\mathbb{R}^{d}\right)$ and $\mathcal{M}_{0, B}\left(\mathbb{R}^{d}\right)$ are invariant subspaces for the semigroup $S(t)$ in $\mathcal{M}_{0}\left(\mathbb{R}^{d}\right)$, which satisfies

$$
\left\|\left|S(t) u_{0}\left\|_{L_{0, B}^{1}\left(\mathbb{R}^{d}\right)} \leq \mid\right\| u_{0} \|_{\mathcal{M}_{0, B}\left(\mathbb{R}^{d}\right)}, \quad t>0\right.\right.
$$

with equality if $u_{0} \geq 0$. For any multi-index $\alpha \in \mathbb{N}^{d}$ there exists a constant $c_{\alpha}>0$ such that

$$
\left\|D_{x}^{\alpha} S(t) u_{0}\right\|_{L_{0, B}^{1}\left(\mathbb{R}^{d}\right)} \leq \frac{c_{\alpha}}{t^{\frac{|\alpha|}{2}}} \mid\left\|u_{0}\right\|_{\mathcal{M}_{0, B}\left(\mathbb{R}^{d}\right)}, \quad t>0 .
$$

In particular $S(t): L_{0, B}^{1}\left(\mathbb{R}^{d}\right) \rightarrow L_{0, B}^{1}\left(\mathbb{R}^{d}\right)$ is an analytic order-preserving contraction semigroup.

See $[4,5]$ for details on analytic semigroups. 


\section{$4 \quad$ An $L_{\varepsilon}^{p}\left(\mathbb{R}^{d}\right)$ theory}

Our goal in this section is to obtain a suitable $L_{\varepsilon}^{p}\left(\mathbb{R}^{d}\right)$ theory for the heat semigroup $S(t)$ from Section 3. For this we define below some suitable weighted $L^{p}$-like spaces and obtain suitable invariance properties as well as suitable $L^{p}-L^{q}$-like estimates. The estimates we obtain reflect the same type of singularities near $t=0$ as the classical estimates in Lebesgue spaces, but, as we are dealing with functions that can become very large at infinity, they differ from the usual estimates for large times in the presence of an additional algebraic growth factor, which (as mentioned in the Introduction) reflects the mechanism of 'mass moving from infinity' described in [6]. Also, it will be necessary to estimate solutions and/or initial data in a different space for each time. This reflects the fact that, as in (2.9), the solution moves with time to a worse space or, as in its equivalent form (3.2), that to estimate the solution in a given space we need an integrability of the initial data that increases with time.

Then recalling the weights in (2.6), for any $1 \leq p<\infty$ we can consider the Banach spaces

$$
L_{\varepsilon}^{p}\left(\mathbb{R}^{d}\right):=\left\{f \in L_{\mathrm{loc}}^{p}\left(\mathbb{R}^{d}\right): \int_{\mathbb{R}^{d}} \rho_{\varepsilon}(x)|f(x)|^{p} \mathrm{~d} x<\infty\right\} ;
$$

with the norm

$$
\|f\|_{L_{\varepsilon}^{p}\left(\mathbb{R}^{d}\right)}:=\left(\int_{\mathbb{R}^{d}} \rho_{\varepsilon}(x)|f(x)|^{p} \mathrm{~d} x\right)^{\frac{1}{p}}
$$

and for $p=\infty$

$$
L_{\varepsilon}^{\infty}\left(\mathbb{R}^{d}\right):=\left\{f \in L_{\mathrm{loc}}^{\infty}\left(\mathbb{R}^{d}\right): \sup _{x \in \mathbb{R}^{d}} \rho_{\varepsilon}(x)|f(x)|<\infty\right\} ;
$$

with the norm

$$
\|f\|_{L_{\varepsilon}^{\infty}\left(\mathbb{R}^{d}\right)}:=\sup _{x \in \mathbb{R}^{d}} \rho_{\varepsilon}(x)|f(x)| .
$$

Also, analogoulsy to (3.1) we define

$$
L_{0}^{p}\left(\mathbb{R}^{d}\right)=\bigcap_{\varepsilon>0} L_{\varepsilon}^{p}\left(\mathbb{R}^{d}\right) \quad \text { and } \quad L_{0}^{\infty}\left(\mathbb{R}^{d}\right)=\bigcap_{\varepsilon>0} L_{\varepsilon}^{\infty}\left(\mathbb{R}^{d}\right) .
$$

These are Fréchet spaces with the corresponding family of norms.

The spaces above have the following inclusion properties.

Lemma 4.1. (i) The spaces $L_{\varepsilon}^{p}\left(\mathbb{R}^{d}\right)$ are increasing in $\varepsilon$ for every $1 \leq p \leq \infty$.

(ii) For $0<\gamma \leq \varepsilon$ and any $1 \leq q \leq p<\infty$ we have

$$
L_{\gamma}^{p}\left(\mathbb{R}^{d}\right) \subset L_{\varepsilon}^{q}\left(\mathbb{R}^{d}\right)
$$

and if $0<\gamma<\varepsilon$ and $1 \leq q \leq \varepsilon / \gamma$ we have

$$
L_{\gamma}^{\infty}\left(\mathbb{R}^{d}\right) \subset L_{\varepsilon}^{q}\left(\mathbb{R}^{d}\right)
$$

with continuous inclusions with norm one. In particular,

$$
L_{\varepsilon}^{p}\left(\mathbb{R}^{d}\right) \subset L_{\varepsilon}^{q}\left(\mathbb{R}^{d}\right) \subset L_{\varepsilon}^{1}\left(\mathbb{R}^{d}\right), \quad 1 \leq q \leq p<\infty .
$$


(iii) Also

$$
L_{0}^{p}\left(\mathbb{R}^{d}\right) \subset L_{0}^{q}\left(\mathbb{R}^{d}\right) \subset L_{0}^{1}\left(\mathbb{R}^{d}\right), \quad 1 \leq q \leq p \leq \infty .
$$

Proof. (i) Notice that (2.7) implies that the spaces $L_{\varepsilon}^{p}\left(\mathbb{R}^{d}\right)$ are increasing in $\varepsilon>0$ for $1 \leq p \leq \infty$.

(ii) If $p>q \geq 1$, then, for a suitable $0<\alpha<1$ to be chosen later, we have

$$
\int_{\mathbb{R}^{d}}|f(x)|^{q} \rho_{\varepsilon}(x) \mathrm{d} x \leq\left[\int_{\mathbb{R}^{d}}|f(x)|^{p} \rho_{\varepsilon}(x)^{\alpha p / q} \mathrm{~d} x\right]^{q / p}\left[\int_{\mathbb{R}^{d}} \rho_{\varepsilon}(x)^{(1-\alpha) p /(p-q)} \mathrm{d} x\right]^{(p-q) / p}
$$

Since $\rho_{\varepsilon}(x)^{p / q}=c \rho_{\varepsilon \alpha p / q}(x)$, we take $0<\alpha<1$ such that $\varepsilon \alpha p / q=\gamma$ which is possible since $\gamma / \varepsilon \leq 1<p / q$.

Also, for $p=\infty$

$$
\int_{\mathbb{R}^{d}}|f(x)|^{q} \rho_{\varepsilon}(x) \mathrm{d} x \leq \int_{\mathbb{R}^{d}}|f(x)|^{q} \mathrm{e}^{-q \gamma|x|^{2}} \rho_{\varepsilon}(x) \mathrm{e}^{q \gamma|x|^{2}} \mathrm{~d} x \leq c\|f\|_{L_{\gamma}^{\infty}\left(\mathbb{R}^{d}\right)}^{q} \int_{\mathbb{R}^{d}} \mathrm{e}^{(q \gamma-\varepsilon)|x|^{2}} \mathrm{~d} x
$$

which is finite, provided that $1 \leq q<\varepsilon / \gamma$.

Taking $\gamma=\varepsilon$ and $\alpha p / q=1$ we obtain

$$
\|f\|_{L_{\varepsilon}^{q}\left(\mathbb{R}^{d}\right)} \leq\|f\|_{L_{\varepsilon}^{p}\left(\mathbb{R}^{d}\right)}\left(\int_{\mathbb{R}^{d}} \rho_{\varepsilon}(x) \mathrm{d} x\right)^{\frac{1}{q}-\frac{1}{p}}=\|f\|_{L_{\varepsilon}^{p}\left(\mathbb{R}^{d}\right)} .
$$

(iii) Follows easily from (ii).

The following result characterizes the complex interpolation spaces between $L_{\varepsilon}^{1}\left(\mathbb{R}^{d}\right)$ and $L_{\varepsilon}^{\infty}\left(\mathbb{R}^{d}\right)$; we will use this result in order to obtain our $L_{p \varepsilon}^{p}-L_{q \varepsilon}^{q}$ estimates on the semigroup $S(t)$. Note that the natural norm on the interpolation space differs from the norm on $L_{p \varepsilon}^{p}\left(\mathbb{R}^{d}\right)$ by a factor that depends on both $p$ and $\varepsilon$.

Lemma 4.2. For $1<p<\infty$ the complex interpolation spaces between $L_{\varepsilon}^{1}\left(\mathbb{R}^{d}\right)$ and $L_{\varepsilon}^{\infty}\left(\mathbb{R}^{d}\right)$ are given by

$$
\left[L_{\varepsilon}^{1}\left(\mathbb{R}^{d}\right), L_{\varepsilon}^{\infty}\left(\mathbb{R}^{d}\right)\right]_{\frac{1}{p^{\prime}}}=\left\{f \in L_{\mathrm{loc}}^{1}\left(\mathbb{R}^{d}\right), \int_{\mathbb{R}^{d}} \rho_{\varepsilon}^{p}(x)|f|^{p}(x) \mathrm{d} x<\infty\right\}=L_{p \varepsilon}^{p}\left(\mathbb{R}^{d}\right)
$$

with norm

$$
\|f\|_{p, \varepsilon}:=\|f\|_{\left[L_{\varepsilon}^{1}\left(\mathbb{R}^{d}\right), L_{\varepsilon}^{\infty}\left(\mathbb{R}^{d}\right)\right]_{\frac{1}{p^{\prime}}}}=\left\|f \rho_{\varepsilon}\right\|_{L^{p}\left(\mathbb{R}^{d}\right)}=\left(\frac{\varepsilon}{\pi}\right)^{\frac{d}{2}\left(1-\frac{1}{p}\right)} \frac{1}{p^{d / 2 p}}\|f\|_{L_{p \varepsilon}^{p}\left(\mathbb{R}^{d}\right)} .
$$

Proof. The operator $\Phi_{\varepsilon}$ defined by setting

$$
\Phi_{\varepsilon}(f):=\rho_{\varepsilon} f
$$

is an isometric isomorphism from $L_{\varepsilon}^{1}\left(\mathbb{R}^{d}\right) \rightarrow L^{1}\left(\mathbb{R}^{d}\right)$ and from $L_{\varepsilon}^{\infty}\left(\mathbb{R}^{d}\right) \rightarrow L^{\infty}\left(\mathbb{R}^{d}\right)$. Thus for any $\theta \in(0,1)$ it follows that

$$
\Phi_{\varepsilon}:\left[L_{\varepsilon}^{1}\left(\mathbb{R}^{d}\right), L_{\varepsilon}^{\infty}\left(\mathbb{R}^{d}\right)\right]_{\theta} \rightarrow\left[L^{1}\left(\mathbb{R}^{d}\right), L^{\infty}\left(\mathbb{R}^{d}\right)\right]_{\theta}
$$


is also an isometric isomorphism.

From the Riesz-Thorin interpolation theorem, see [1], we have

$$
\left[L^{1}\left(\mathbb{R}^{d}\right), L^{\infty}\left(\mathbb{R}^{d}\right)\right]_{1 / p^{\prime}}=L^{p}\left(\mathbb{R}^{d}\right), \quad 1<p<\infty,
$$

and since $\rho_{\varepsilon}^{p}(x)=c_{\varepsilon, p} \rho_{p \varepsilon}(x)$, with $c_{\varepsilon, p}=(\varepsilon / \pi)^{\frac{d}{2}(p-1)} p^{-d / 2}$, we obtain (4.3) and (4.4).

Remark 4.3. From Lemma A.1 in [6] the operator $\Phi_{\varepsilon}$ in the proof of Lemma 4.2 can be defined for measures such that for $\mu \in \mathcal{M}_{\varepsilon}\left(\mathbb{R}^{d}\right)$ and for any borel measurable set $A \subset \mathbb{R}^{d}$ we have

$$
\Phi_{\varepsilon}(\mu)(A)=\int_{A} \rho_{\varepsilon}(x) \mathrm{d} \mu(x),
$$

that is, $\mathrm{d} \Phi_{\varepsilon}(\mu)=\rho_{\varepsilon} \mathrm{d} \mu$. Therefore in this setting we have $\Phi_{\varepsilon}: \mathcal{M}_{\varepsilon}\left(\mathbb{R}^{d}\right) \rightarrow \mathcal{M}_{\mathrm{BTV}}\left(\mathbb{R}^{d}\right)=$ $\left(C_{0}\left(\mathbb{R}^{d}\right)\right)^{\prime}$, the space of measures of bounded total variation, is also an isometric isomorphism.

Finally, defining

$$
C_{\varepsilon, 0}\left(\mathbb{R}^{d}\right)=\left\{f \in L_{\mathrm{loc}}^{1}\left(\mathbb{R}^{d}\right): \rho_{\varepsilon} f \in C_{0}\left(\mathbb{R}^{d}\right)\right\} \subset L_{\varepsilon}^{\infty}\left(\mathbb{R}^{d}\right)
$$

with the norm (4.2), then $\Phi_{\varepsilon}: C_{\varepsilon, 0}\left(\mathbb{R}^{d}\right) \rightarrow C_{0}\left(\mathbb{R}^{d}\right)$ is also an isometric isomorphism.

In what follows we denote by $S(t)$ the map from $\mathcal{M}_{0}\left(\mathbb{R}^{d}\right)$ into $L_{0}^{1}\left(\mathbb{R}^{d}\right)$ introduced in Proposition 3.3. We start by proving the following estimates of " $L^{1}-L^{\infty}$ " and " $L^{\infty}-L^{\infty}$ " type.

Proposition 4.4. If $u_{0} \in \mathcal{M}_{0}\left(\mathbb{R}^{d}\right)$ then $u(t)=S(t) u_{0}$ given by (2.2) satisfies the following estimates for any $\delta>0, t>0$ and $\delta(t)=\frac{\delta}{1+4 \delta t}$.

(i) If $u_{0} \in \mathcal{M}_{0}\left(\mathbb{R}^{d}\right)$ then $u(t) \in L_{0}^{\infty}\left(\mathbb{R}^{d}\right)$ for all $t>0$ and

$$
\|u(t)\|_{L_{\delta}^{\infty}\left(\mathbb{R}^{d}\right)} \leq\left(\frac{1+4 \delta t}{4 \pi t}\right)^{d / 2}\left\|u_{0}\right\|_{\mathcal{M}_{\delta(t)}\left(\mathbb{R}^{d}\right)} .
$$

(ii) If $u_{0} \in L_{0}^{\infty}\left(\mathbb{R}^{d}\right)$ then $u(t) \in L_{0}^{\infty}\left(\mathbb{R}^{d}\right)$ for all $t>0$ and

$$
\|u(t)\|_{L_{\delta}^{\infty}\left(\mathbb{R}^{d}\right)} \leq(1+4 \delta t)^{d}\left\|u_{0}\right\|_{L_{\delta(t)}^{\infty}\left(\mathbb{R}^{d}\right)} .
$$

Proof. Setting $0<\delta=\frac{1}{4 \tau}$, from (2.2) we have

$$
\mathrm{e}^{-\delta|x|^{2}}|u(x, t)| \leq \frac{1}{(4 \pi t)^{d / 2}} \int_{\mathbb{R}^{d}} \mathrm{e}^{-|x|^{2} / 4 \tau} \mathrm{e}^{-|x-z|^{2} / 4 t} \mathrm{~d}\left|u_{0}(z)\right| .
$$

Notice that completing squares as

$$
\frac{|x|^{2}}{\tau}+\frac{|x-z|^{2}}{t}=\frac{t+\tau}{t \tau}\left|x-\frac{\tau}{t+\tau} z\right|^{2}+\frac{|z|^{2}}{t+\tau}
$$


we get

$$
\mathrm{e}^{-\delta|x|^{2}}|u(x, t)| \leq \frac{1}{(4 \pi t)^{d / 2}} \int_{\mathbb{R}^{d}} \mathrm{e}^{-\frac{t+\tau}{4 t \tau}\left|x-\frac{\tau}{t+\tau} z\right|^{2}} \mathrm{e}^{-\frac{|z|^{2}}{4(t+\tau)}} \mathrm{d}\left|u_{0}(z)\right|
$$

(i) First, from (4.8) we get

$$
\mathrm{e}^{-\delta|x|^{2}}|u(x, t)| \leq \frac{1}{(4 \pi t)^{d / 2}} \int_{\mathbb{R}^{d}} e^{-\frac{|z|^{2}}{4(t+\tau)}} \mathrm{d}\left|u_{0}(z)\right|
$$

Therefore, setting $\delta(t)=\frac{1}{4(t+\tau)}=\frac{\delta}{1+4 \delta t}$, we get

$$
\sup _{x \in \mathbb{R}^{d}} \mathrm{e}^{-\delta|x|^{2}}|u(x, t)| \leq \frac{1}{(4 \pi t)^{d / 2}}\left(\frac{\pi}{\delta(t)}\right)^{d / 2}\left\|u_{0}\right\|_{\mathcal{M}_{\delta(t)}\left(\mathbb{R}^{d}\right)}
$$

Hence

$$
\|u(t)\|_{L_{\delta}^{\infty}\left(\mathbb{R}^{d}\right)} \leq\left(\frac{\delta}{\pi}\right)^{d / 2} \frac{1}{(4 \pi t)^{d / 2}}\left(\frac{\pi}{\delta(t)}\right)^{d / 2}\left\|u_{0}\right\|_{\mathcal{M}_{\delta(t)}\left(\mathbb{R}^{d}\right)}=\frac{1}{(4 \pi t)^{d / 2}}\left(\frac{\delta}{\delta(t)}\right)^{d / 2}\left\|u_{0}\right\|_{\mathcal{M}_{\delta(t)}\left(\mathbb{R}^{d}\right)}
$$

and we get (4.5).

(ii) From (4.8) we also get

$$
\mathrm{e}^{-\delta|x|^{2}}|u(x, t)| \leq \frac{1}{(4 \pi t)^{d / 2}}\left(\int_{\mathbb{R}^{d}} \mathrm{e}^{-\frac{t+\tau}{4 t \tau}\left|x-\frac{\tau}{t+\tau} z\right|^{2}} \mathrm{~d} z\right) \sup _{z \in \mathbb{R}^{d}} \mathrm{e}^{-\frac{|z|^{2}}{4(t+\tau)}}\left|u_{0}(z)\right| .
$$

Now, setting again $\delta(t)=\frac{1}{4(t+\tau)}=\frac{\delta}{1+4 \delta t}$, we get

$$
\int_{\mathbb{R}^{d}} \mathrm{e}^{-\frac{t+\tau}{4 t \tau}\left|x-\frac{\tau}{t+\tau} z\right|^{2}} \mathrm{~d} z=\int_{\mathbb{R}^{d}} \mathrm{e}^{-\frac{\tau}{4 t(t+\tau)}|z|^{2}} \mathrm{~d} z=\left(\frac{4 \pi t(t+\tau)}{\tau}\right)^{d / 2}=\left(\frac{4 \pi t \delta}{\delta(t)}\right)^{d / 2}=(4 \pi t(1+4 \delta t))^{d / 2}
$$

which leads to

$$
\sup _{x \in \mathbb{R}^{d}} \mathrm{e}^{-\delta|x|^{2}}|u(x, t)| \leq \frac{1}{(4 \pi t)^{d / 2}}(4 \pi t(1+4 \delta t))^{d / 2}\left(\frac{\pi}{\delta(t)}\right)^{d / 2}\left\|u_{0}\right\|_{\mathcal{M}_{\delta(t)}\left(\mathbb{R}^{d}\right)} .
$$

Therefore

$$
\|u(t)\|_{L_{\delta}^{\infty}\left(\mathbb{R}^{d}\right)} \leq\left(\frac{\delta}{\pi}\right)^{d / 2}(1+4 \delta t)^{d / 2}\left(\frac{\pi}{\delta(t)}\right)^{d / 2}\left\|u_{0}\right\|_{\mathcal{M}_{\delta(t)}\left(\mathbb{R}^{d}\right)}
$$

and we get (4.6).

The estimates above and the " $L^{1}-L^{1}$ " bounds in $(3.2)$ will yield the general " $L^{p}-L^{q}$ " bounds via interpolation. However, first we improve the regularity estimates in Proposition 3.3 for initial data in $\mathcal{M}_{0}\left(\mathbb{R}^{d}\right)$.

Proposition 4.5. If $u_{0} \in \mathcal{M}_{0}\left(\mathbb{R}^{d}\right)$ then $u(t)=S(t) u_{0}$ given by $(2.2)$ satisfies

$$
\|u(t)\|_{L_{q \delta}^{q}\left(\mathbb{R}^{d}\right)} \leq q^{d / 2 q}\left(\frac{1+4 \delta t}{4 \delta t}\right)^{\frac{d}{2}\left(1-\frac{1}{q}\right)}\left\|u_{0}\right\|_{\mathcal{M}_{\delta(t)}\left(\mathbb{R}^{d}\right)}
$$


for any $1 \leq q \leq \infty, \delta>0, t>0$, where and

$$
\delta(t):=\frac{\delta}{1+4 \delta t} .
$$

In particular, $S(t): \mathcal{M}_{0}\left(\mathbb{R}^{d}\right) \longrightarrow L_{0}^{q}\left(\mathbb{R}^{d}\right)$ is continuous.

Proof. Interpolate (3.2) and (4.5) to get

$$
\|u(t)\|_{q, \delta} \leq\left(\frac{1+4 \delta t}{4 \pi t}\right)^{d / 2 q^{\prime}}\left\|u_{0}\right\|_{\mathcal{M}_{\delta(t)}\left(\mathbb{R}^{d}\right)} .
$$

Then use (4.4) to deduce that

$$
\left(\frac{\delta}{\pi}\right)^{\frac{d}{2}\left(1-\frac{1}{q}\right)} \frac{1}{q^{d / 2 q}}\|u(t)\|_{L_{q \delta}^{q}\left(\mathbb{R}^{d}\right)} \leq\left(\frac{1+4 \delta t}{4 \pi t}\right)^{d / 2 q^{\prime}}\left\|u_{0}\right\|_{\mathcal{M}_{\delta(t)}\left(\mathbb{R}^{d}\right)} .
$$

that leads to (4.9).

Now we obtain the corresponding regularity estimates assuming the initial data is in $L_{0}^{p}\left(\mathbb{R}^{d}\right)$.

Theorem 4.6. If $u_{0} \in L_{0}^{1}\left(\mathbb{R}^{d}\right)$ then $u(t)=S(t) u_{0}$ given by (2.2) satisfies the following estimates. If $u_{0} \in L_{0}^{p}\left(\mathbb{R}^{d}\right)$ with $1 \leq p<\infty$ and for any $1 \leq p \leq q<\infty$, then $u(t) \in L_{0}^{q}\left(\mathbb{R}^{d}\right)$ for all $t>0$ and for any $\delta>0, t>0$ and $\delta(t)=\frac{\delta}{1+4 \delta t}$

$$
\|u(t)\|_{L_{q \delta}^{q}\left(\mathbb{R}^{d}\right)} \leq c_{p, q} \frac{1}{(4 \delta t)^{\frac{d}{2}\left(\frac{1}{p}-\frac{1}{q}\right)}}(1+4 \delta t)^{\frac{d}{2}\left(1-\frac{1}{q}\right)}\left\|u_{0}\right\|_{L_{p \delta(t)}^{p}\left(\mathbb{R}^{d}\right)}
$$

with $c_{p, q}=\frac{q^{d / 2 q}}{p^{d / 2 p}}$. For $q=\infty$ we have

$$
\|u(t)\|_{L_{\delta}^{\infty}\left(\mathbb{R}^{d}\right)} \leq c_{p, \infty} \frac{1}{(4 \pi t)^{\frac{d}{2 p}}}(1+4 \delta t)^{\frac{d}{2}}\left\|u_{0}\right\|_{L_{p \delta(t)}^{p}}\left(\mathbb{R}^{d}\right)
$$

with $c_{p, \infty}=\left(\frac{\delta}{\pi}\right)^{\frac{d}{2}\left(1-\frac{1}{p}\right)} \frac{1}{p^{d / 2 p}}$.

In particular, $S(t): L_{0}^{p}\left(\mathbb{R}^{d}\right) \longrightarrow L_{0}^{q}\left(\mathbb{R}^{d}\right)$ is continuous.

Proof. (i) Notice that (3.2) for $u_{0} \in L_{0}^{1}\left(\mathbb{R}^{d}\right)$ reads $\|u(t)\|_{L_{\delta}^{1}\left(\mathbb{R}^{d}\right)} \leq\left\|u_{0}\right\|_{L_{\delta(t)}^{1}\left(\mathbb{R}^{d}\right)}$, By interpolating this estimate and (4.6) with $\theta=\frac{1}{p^{\prime}}$

$$
\|u(t)\|_{p, \delta} \leq(1+4 \delta t)^{d / p^{\prime}}\left\|u_{0}\right\|_{p, \delta(t)}
$$

with $\delta(t)=\frac{\delta}{1+4 \delta t}$.

(ii) Interpolating (4.5) for $u_{0} \in L_{0}^{1}\left(\mathbb{R}^{d}\right)$ and (4.6) gives

$$
\|u(t)\|_{L_{\delta}^{\infty}\left(\mathbb{R}^{d}\right)} \leq\left(\frac{1+4 \delta t}{4 \pi t}\right)^{d / 2 p}(1+4 \delta t)^{d / p^{\prime}}\left\|u_{0}\right\|_{p, \delta(t)}=\frac{(1+4 \delta t)^{\frac{d}{2}\left(1+\frac{1}{p^{\prime}}\right)}}{(4 \pi t)^{d / 2 p}}\left\|u_{0}\right\|_{p, \delta(t)} .
$$


Then use (4.4) to get

$$
\|u(t)\|_{L_{\delta}^{\infty}\left(\mathbb{R}^{d}\right)} \leq \frac{(1+4 \delta t)^{\frac{d}{2}\left(1+\frac{1}{p^{\prime}}\right)}}{(4 \pi t)^{d / 2 p}}\left(\frac{\delta(t)}{\pi}\right)^{\frac{d}{2}\left(1-\frac{1}{p}\right)} \frac{1}{p^{d / 2 p}}\left\|u_{0}\right\|_{L_{p \delta(t)}^{p}\left(\mathbb{R}^{d}\right)}
$$

and (4.11) follows.

(iii) Interpolating (4.12) and (4.13) we get for any $0<\theta<1$,

$$
\|u(t)\|_{\left[L_{p \delta}^{p}\left(\mathbb{R}^{d}\right), L_{\delta}^{\infty}\left(\mathbb{R}^{d}\right)\right]_{\theta}} \leq\left(\frac{1+4 \delta t}{4 \pi t}\right)^{d \theta / 2 p}(1+4 \delta t)^{d \theta / p^{\prime}}(1+4 \delta t)^{d(1-\theta) / p^{\prime}}\left\|u_{0}\right\|_{p, \delta(t)}
$$

which gives

$$
\|u(t)\|_{\left[L_{p \delta}^{p}\left(\mathbb{R}^{d}\right), L_{\delta}^{\infty}\left(\mathbb{R}^{d}\right)\right]_{\theta}} \leq\left(\frac{1+4 \delta t}{4 \pi t}\right)^{d \theta / 2 p}(1+4 \delta t)^{d / p^{\prime}}\left\|u_{0}\right\|_{p, \delta(t)} .
$$

By reiteration of the interpolation argument in (4.3) we have that for $1-\theta=\frac{p}{q}$, with $q>p$ we get

$$
\left[L_{p \delta}^{p}\left(\mathbb{R}^{d}\right), L_{\delta}^{\infty}\left(\mathbb{R}^{d}\right)\right]_{\theta}=L_{q \delta}^{q}\left(\mathbb{R}^{d}\right) .
$$

Indeed as in Lemma 4.2 we have that $\Phi_{\delta}$ is also an isometric isomorphism between $L_{p \delta}^{p}\left(\mathbb{R}^{d}\right)$ and $L^{p}\left(\mathbb{R}^{d}\right)$ and between $L_{\delta}^{\infty}\left(\mathbb{R}^{d}\right)$ and $L^{\infty}\left(\mathbb{R}^{d}\right)$. Since for $q>p$ and $1-\theta=\frac{p}{q}$ we ave

$$
\left[L^{p}\left(\mathbb{R}^{d}\right), L^{\infty}\left(\mathbb{R}^{d}\right)\right]_{\theta}=L^{q}\left(\mathbb{R}^{d}\right)
$$

we get the claim.

This leads to the estimate

$$
\|u(t)\|_{q, \delta} \leq\left(\frac{1+4 \delta t}{4 \pi t}\right)^{\frac{d}{2}\left(\frac{1}{p}-\frac{1}{q}\right)}(1+4 \delta t)^{\frac{d}{p^{\prime}}}\left\|u_{0}\right\|_{p, \delta(t)}=\frac{(1+4 \delta t)^{\frac{d}{2}\left(\frac{1}{p^{\prime}}+\frac{1}{q^{\prime}}\right)}}{(4 \pi t)^{\frac{d}{2}\left(\frac{1}{p}-\frac{1}{q}\right)}}\left\|u_{0}\right\| \|_{p, \delta(t)} .
$$

From (4.14) and (4.4) we get

$$
\left(\frac{\delta}{\pi}\right)^{\frac{d}{2}\left(1-\frac{1}{q}\right)} \frac{1}{q^{d / 2 q}}\|u(t)\|_{L_{q \delta}^{q}\left(\mathbb{R}^{d}\right)} \leq \frac{(1+4 \delta t)^{\frac{d}{2}\left(\frac{1}{p^{\prime}}+\frac{1}{q^{\prime}}\right)}}{(4 \pi t)^{\frac{d}{2}\left(\frac{1}{p}-\frac{1}{q}\right)}}\left(\frac{\delta(t)}{\pi}\right)^{\frac{d}{2}\left(1-\frac{1}{p}\right)} \frac{1}{p^{d / 2 p}}\left\|u_{0}\right\|_{L_{p \delta(t)}^{p}}\left(\mathbb{R}^{d}\right)
$$

with $\delta(t)=\frac{\delta}{1+4 \delta t}$ which leads to (4.10).

We can also obtain the following bounds on the derivatives of the solutions.

Proposition 4.7. If $u_{0} \in \mathcal{M}_{0}\left(\mathbb{R}^{d}\right)$ then $u(t)=S(t) u_{0}$ given by (2.2) satisfies the following estimates.

(i) For any multi-index $\alpha \in \mathbb{N}^{d}$ and $1 \leq q<\infty$, then for any $\delta>0, t>0, \gamma>1$ and $\tilde{\delta}(t)=\frac{\delta}{1+4 \gamma \delta t}$

$$
\left\|D_{x}^{\alpha} u(t)\right\|_{L_{q \delta}^{q}\left(\mathbb{R}^{d}\right)} \leq \frac{c_{\alpha, q, \delta, \gamma}}{t^{\frac{|\alpha|}{2}}}\left(\frac{1+4 \gamma \delta t}{t}\right)^{\frac{d}{2}\left(1-\frac{1}{q}\right)}\left\|u_{0}\right\|_{\mathcal{M}_{\tilde{\delta}(t)}\left(\mathbb{R}^{d}\right)}
$$


and

$$
\left\|D_{x}^{\alpha} u(t)\right\|_{L_{\delta}^{\infty}\left(\mathbb{R}^{d}\right)} \leq \frac{c_{\alpha, q, \delta, \gamma}}{t^{\frac{|\alpha|}{2}}}\left(\frac{1+4 \gamma \delta t}{t}\right)^{\frac{d}{2}}\left\|u_{0}\right\|_{\mathcal{M}_{\tilde{\delta}(t)}\left(\mathbb{R}^{d}\right)} .
$$

(ii) If $u_{0} \in L_{0}^{p}\left(\mathbb{R}^{d}\right)$ with $1 \leq p \leq \infty$ for any multi-index $\alpha \in \mathbb{N}^{d}$ and for any $1 \leq p \leq q \leq \infty$, then for any $\delta>0, t>0, \gamma>1$ and $\tilde{\delta}(t)=\frac{\delta}{1+4 \gamma \delta t}$

$$
\left\|D_{x}^{\alpha} u(t)\right\|_{L_{q \delta}^{q}\left(\mathbb{R}^{d}\right)} \leq \frac{c_{\alpha, p, q, \delta, \gamma}}{t^{\frac{|\alpha|}{2}+\frac{d}{2}\left(\frac{1}{p}-\frac{1}{q}\right)}}(1+4 \gamma \delta t)^{\frac{d}{2}\left(1-\frac{1}{q}\right)}\left\|u_{0}\right\|_{L_{p \tilde{\delta}(t)}^{p}\left(\mathbb{R}^{d}\right)}
$$

and

$$
\left\|D_{x}^{\alpha} u(t)\right\|_{L_{\delta}^{\infty}\left(\mathbb{R}^{d}\right)} \leq \frac{c_{\alpha, p, \delta, \gamma}}{t^{\frac{|\alpha|}{2}+\frac{d}{2 p}}}(1+4 \gamma \delta t)^{\frac{d}{2}}\left\|u_{0}\right\|_{L_{p \tilde{\delta}(t)}^{p}\left(\mathbb{R}^{d}\right)} .
$$

Proof. Using (2.11) in Remark 2.3 we obtain,

$$
\mathrm{e}^{-|x|^{2} / 4 \tau}\left|D_{x}^{\alpha} u(x, t)\right| \leq \frac{c_{\alpha, \beta}}{t^{d / 2+|\alpha| / 2}} \int_{\mathbb{R}^{d}} \mathrm{e}^{-|x|^{2} / 4 \tau} \mathrm{e}^{-(1-\beta) \frac{|x-y|^{2}}{4 t}} \mathrm{~d}\left|u_{0}(z)\right| .
$$

The integral term in the right hand side above is like (4.7) but with the extra factor $\frac{1}{t^{\frac{|\alpha|}{2}}}$ and $t^{\prime}=\gamma t$, with $\gamma=\frac{1}{1-\beta}>1$, replacing $t$ in the integral term. Therefore we can proceed first as in the proof of Proposition 4.4 and obtain from (4.17) the estimates in there with the right hand side in terms of $t^{\prime}=\gamma t$ and with $\tilde{\delta}(t)=\frac{\delta}{1+4 \delta t^{\prime}}$.

(i) In particular, we get

$$
\left\|D_{x}^{\alpha} u(t)\right\|_{L_{\delta}^{\infty}\left(\mathbb{R}^{d}\right)} \leq \frac{c_{\alpha, \gamma}}{t^{\frac{|\alpha|}{2}}}\left(\frac{1+4 \gamma \delta t}{4 \pi t}\right)^{d / 2}\left\|u_{0}\right\|_{\mathcal{M}_{\tilde{\delta}(t)}\left(\mathbb{R}^{d}\right)} .
$$

Interpolating this and (3.3) gives

$$
\left\|D_{x}^{\alpha} u(t)\right\|_{q, \delta} \leq \frac{c}{t^{\frac{|\alpha|}{2}}}\left(\frac{1+4 \gamma \delta t}{4 \pi t}\right)^{d / 2 q^{\prime}}\left\|u_{0}\right\|_{\mathcal{M}_{\tilde{\delta}(t)}}\left(\mathbb{R}^{d}\right) .
$$

Then use (4.4) to get

$$
\left(\frac{\delta}{\pi}\right)^{\frac{d}{2}\left(1-\frac{1}{q}\right)} \frac{1}{q^{d / 2 q}}\left\|D_{x}^{\alpha} u(t)\right\|_{L_{q \delta}^{q}\left(\mathbb{R}^{d}\right)} \leq \frac{c}{t^{\frac{|\alpha|}{2}}}\left(\frac{1+4 \gamma \delta t}{4 \pi t}\right)^{d / 2 q^{\prime}}\left\|u_{0}\right\|_{\mathcal{M}_{\tilde{\delta}(t)}\left(\mathbb{R}^{d}\right)}
$$

which leads to (4.15).

(ii) After the estimates as in Proposition 4.4 sketched above, we can proceed as in Theorem 4.6 (using (3.3)) and obtain from (4.17) all estimates in there with the right hand side in terms of $t^{\prime}=\gamma t$ and with $\tilde{\delta}(t)=\frac{\delta}{1+4 \delta t^{\prime}}$. Writing everything in terms of $t$ we get, analogously to (4.11)

$$
\left\|D_{x}^{\alpha} u(t)\right\|_{L_{\delta}^{\infty}\left(\mathbb{R}^{d}\right)} \leq c_{\alpha} \frac{1}{t^{\frac{d}{2 p}}}(1+4 \gamma \delta t)^{\frac{d}{2}}\left\|u_{0}\right\|_{L_{p \tilde{\delta}(t)}^{p}\left(\mathbb{R}^{d}\right)}
$$


and analogously to (4.14)

$$
\left\|D_{x}^{\alpha} u(t)\right\|_{q, \delta} \leq \frac{c_{\alpha}}{t^{\frac{|\alpha|}{2}}} \frac{(1+4 \gamma \delta t)^{\frac{d}{2}\left(\frac{1}{p^{\prime}}+\frac{1}{q^{\prime}}\right)}}{t^{\frac{d}{2}\left(\frac{1}{p}-\frac{1}{q}\right)}}\left\|u_{0}\right\|_{p, \tilde{\delta}(t)} .
$$

Again, using the norms (4.1) and (4.4) we get the result.

Note that Theorem 4.6 ensures the invariance of $L_{0}^{p}\left(\mathbb{R}^{d}\right)$ under the semigroup $S(t)$. This combined with Proposition 4.7 yields the following.

Proposition 4.8. Assume that $1 \leq p<\infty$. For $u_{0} \in L_{0}^{p}\left(\mathbb{R}^{d}\right)$ the family of mappings defined by

$$
u(t)=S(t) u_{0}, \quad t \geq 0,
$$

as in $(2.2)$, satisfy $S(t): L_{0}^{p}\left(\mathbb{R}^{d}\right) \longrightarrow L_{0}^{p}\left(\mathbb{R}^{d}\right)$, are linear, continuous, and order preserving and $\{S(t)\}_{t \geq 0}$ is a semigroup in $L_{0}^{p}\left(\mathbb{R}^{d}\right)$. Also for any multi-index $\alpha \in \mathbb{N}^{d} \cup\{0\}$, any $p \leq q \leq \infty$, and $t>0, D_{x}^{\alpha} S(t): L_{0}^{p}\left(\mathbb{R}^{d}\right) \longrightarrow L_{0}^{q}\left(\mathbb{R}^{d}\right)$ is linear and continuous.

Proof. From Proposition 3.3 and Theorem 4.6 with $q=p$ and Proposition 4.7 it only remains to prove the continuity at $t=0$. For this note that for any $\varphi \in C_{c}\left(\mathbb{R}^{d}\right)$ we have for $\delta>0$

$$
\left\|S(t) u_{0}-u_{0}\right\|_{L_{p \delta}^{p}\left(\mathbb{R}^{d}\right)} \leq\left\|S(t) u_{0}-S(t) \varphi\right\|_{L_{p \delta}^{p}\left(\mathbb{R}^{d}\right)}+\|S(t) \varphi-\varphi\|_{L_{p \delta}^{p}\left(\mathbb{R}^{d}\right)}+\left\|\varphi-u_{0}\right\|_{L_{p \delta}^{p}\left(\mathbb{R}^{d}\right)} .
$$

Let $\gamma>0$ and take $\varphi \in C_{c}\left(\mathbb{R}^{d}\right)$ such that

$$
\left\|u_{0}-\varphi\right\|_{L_{\varepsilon}^{p}\left(\mathbb{R}^{d}\right)}^{p}=\int_{\mathbb{R}^{\mathrm{d}}} \mathrm{e}^{-\varepsilon|x|^{2}}\left|u_{0}(x)-\varphi(x)\right|^{p} \mathrm{~d} x<\gamma^{p} .
$$

To see this note that for $R>0$, if $\operatorname{supp}(\varphi) \subset B(0, R)$ then

$$
\int_{\mathbb{R}^{\mathrm{d}}} \mathrm{e}^{-\varepsilon|x|^{2}}\left|u_{0}(x)-\varphi(x)\right|^{p} \mathrm{~d} x=\int_{|x| \leq R} \mathrm{e}^{-\varepsilon|x|^{2}}\left|u_{0}(x)-\varphi(x)\right|^{p} \mathrm{~d} x+\int_{|x|>R} \mathrm{e}^{-\varepsilon|x|^{2}}\left|u_{0}(x)\right|^{p} \mathrm{~d} x .
$$

The second term is small for $R$ large and so is the first one if we approach $u_{0}$ by $\varphi$ in $L^{p}(B(0, R))$.

Now for any $\delta>\varepsilon$ and all sufficiently small $t$ we get $\varepsilon<\delta(t)=\frac{\delta}{1+4 \delta t}<\delta$. Then from (4.10) with $q=p$ we have

$$
\begin{aligned}
\left\|S(t)\left(u_{0}-\varphi\right)\right\|_{L_{p \delta}^{p}\left(\mathbb{R}^{d}\right)} & \leq c\left(\frac{\delta}{\varepsilon}\right)^{d / 2 p}\left\|S(t)\left(u_{0}-\varphi\right)\right\|_{L_{p \delta(t)}^{p}\left(\mathbb{R}^{d}\right)} \\
& \leq c\left(\frac{\delta}{\varepsilon}\right)^{d / 2 p}\left\|u_{0}-\varphi\right\|_{L_{p \varepsilon}^{p}\left(\mathbb{R}^{d}\right)}<c\left(\frac{\delta}{\varepsilon}\right)^{d / 2 p} \gamma .
\end{aligned}
$$

Finally, we know that $S(t) \varphi-\varphi \rightarrow 0$ uniformly in $\mathbb{R}^{d}$ as $t \rightarrow 0$. Hence $\|S(t) \varphi-\varphi\|_{L_{p \delta}^{p}\left(\mathbb{R}^{d}\right)} \rightarrow 0$ as $t \rightarrow 0$, which proves the required continuity at $t=0$.

Now we improve the regularity of the solution curves in Proposition 3.5. 
Proposition 4.9. For each $u_{0} \in \mathcal{M}_{0}\left(\mathbb{R}^{d}\right)$, any $1 \leq p<\infty$, and any multi-index $\alpha \in \mathbb{N}^{d}$, the solution curve

$$
(0, \infty) \ni t \longmapsto D_{x}^{\alpha} S(t) u_{0} \in L_{0}^{p}\left(\mathbb{R}^{d}\right)
$$

is $C^{\infty}$. If additionally, $u_{0} \in \mathcal{M}_{0, B}\left(\mathbb{R}^{d}\right)$ as in (3.8), that is

$$
\sup _{\varepsilon>0}\left\|u_{0}\right\|_{\mathcal{M}_{\varepsilon}\left(\mathbb{R}^{d}\right)}<\infty
$$

then the solution curve is $C^{\omega}\left((0, \infty), L_{0}^{p}\left(\mathbb{R}^{d}\right)\right)$, i.e. analytic.

Proof. From Proposition 3.3 we can assume that $u_{0} \in L_{0}^{1}\left(\mathbb{R}^{d}\right)$. Otherwise, we use the semigroup property and for any sufficiently small $\varepsilon>0$, consider initial data $S(\varepsilon) u_{0} \in L_{0}^{1}\left(\mathbb{R}^{d}\right)$

Now for $u_{0} \in L_{0}^{1}\left(\mathbb{R}^{d}\right)$, fix $\varepsilon>0$ and $t>0$ and a multi-index $\alpha \in \mathbb{N}^{d}$, write

$$
D_{x}^{\alpha} S(t+h) u_{0}-D_{x}^{\alpha} S(t) u_{0}=D_{x}^{\alpha} S(t-\varepsilon)\left(S(\varepsilon+h) u_{0}-S(\varepsilon) u_{0}\right) \rightarrow 0, \quad h \rightarrow 0,
$$

since from Proposition 3.5 the term in parentheses converges to 0 in $L_{0}^{1}\left(\mathbb{R}^{d}\right)$ and, from (4.16), $D_{x}^{\alpha} S(t-\varepsilon)$ is continuous from $L_{0}^{1}\left(\mathbb{R}^{d}\right)$ to $L_{0}^{p}\left(\mathbb{R}^{d}\right)$.

If additionally $u_{0}$ satisfies (3.4) then from Proposition 3.5 for any fixed $\gamma>0$ and $t_{0}>0$ the Taylor series

$$
u(t)=S(t) u_{0}=\sum_{m=0}^{\infty} \frac{1}{m !} u^{m)}\left(t_{0}\right)\left(t-t_{0}\right)^{m}, \quad \text { with } \quad u^{m)}\left(t_{0}\right)=S^{m)}\left(t_{0}\right) u_{0}
$$

has a positive radius of convergence in $L_{\gamma}^{1}\left(\mathbb{R}^{d}\right)$. Also, from (3.7), for any $\varepsilon>0$,

$$
u^{m)}\left(t_{0}\right)=(-\Delta)^{m} S\left(t_{0}\right) u_{0}=S(\varepsilon)(-\Delta)^{m} S\left(t_{0}-\varepsilon\right) u_{0} .
$$

Hence, from (4.10) we get for $\delta>0$ and $\gamma=\frac{\delta}{1+4 \delta \varepsilon}$

$$
\left\|u^{m)}\left(t_{0}\right)\right\|_{L_{p \delta}^{p}\left(\mathbb{R}^{d}\right)} \leq C(\varepsilon)\left\|(-\Delta)^{m} S\left(t_{0}-\varepsilon\right) u_{0}\right\|_{L_{\gamma}^{1}\left(\mathbb{R}^{d}\right)}=C(\varepsilon)\left\|u^{m)}\left(t_{0}-\varepsilon\right)\right\|_{L_{\gamma}^{1}\left(\mathbb{R}^{d}\right)}, \quad m \in \mathbb{N}
$$

and so the Taylor series has a positive radius of convergence in $L_{p \delta}^{p}\left(\mathbb{R}^{d}\right)$ at least as large as that in $L_{\gamma}^{1}\left(\mathbb{R}^{d}\right)$.

Notice that all the estimates we have obtained so far in this section have been for initial data in the spaces $L_{0}^{p}\left(\mathbb{R}^{d}\right), 1 \leq p \leq \infty$, or $\mathcal{M}_{0}\left(\mathbb{R}^{d}\right)$.

Now we will use the fact that for $1 \leq p<\infty$ the space $L_{0}^{p}\left(\mathbb{R}^{d}\right)$ is dense in all spaces $L_{\varepsilon}^{p}\left(\mathbb{R}^{d}\right)$ for $\varepsilon>0$ and $\mathcal{M}_{0}\left(\mathbb{R}^{d}\right)$ is dense in $\mathcal{M}_{\varepsilon}\left(\mathbb{R}^{d}\right)$ for $\varepsilon>0$. The proof of this fact will be deferred to Section 6 where it will be proved in a broader context; see Lemma 6.2 and Lemma 6.3. Given this, a density argument allows us to extend the estimates above to initial data in the spaces $L_{\varepsilon}^{p}\left(\mathbb{R}^{d}\right)$, where solutions of the heat equation may exist only for finite time, see [6]. In particular, we extend estimates (3.2), (4.5), (4.6), (4.9), (4.10) and (4.11).

Proposition 4.10. Assume that $\varepsilon>0$ and $T(\varepsilon)=\frac{1}{4 \varepsilon}$. For every $\delta>\varepsilon$ take $0 \leq t \leq \frac{1}{4 \varepsilon}-\frac{1}{4 \delta}=$ $T(\varepsilon)-T(\delta)$. 
(i) For any $u_{0} \in \mathcal{M}_{\varepsilon}\left(\mathbb{R}^{d}\right)$ we have

$$
\|u(t)\|_{L_{\delta}^{1}\left(\mathbb{R}^{d}\right)} \leq\left\|u_{0}\right\|_{\mathcal{M}_{\delta(t)}\left(\mathbb{R}^{d}\right)},
$$

with equality if $u_{0} \geq 0$, and also

$$
\begin{gathered}
\|u(t)\|_{L_{\delta}^{\infty}\left(\mathbb{R}^{d}\right)} \leq\left(\frac{1+4 \delta t}{4 \pi t}\right)^{d / 2}\left\|u_{0}\right\|_{\mathcal{M}_{\delta(t)}\left(\mathbb{R}^{d}\right)} \\
\|u(t)\|_{L_{q \delta}^{q}\left(\mathbb{R}^{d}\right)} \leq q^{d / 2 q}\left(\frac{1+4 \delta t}{4 \delta t}\right)^{\frac{d}{2}\left(1-\frac{1}{q}\right)}\left\|u_{0}\right\|_{\mathcal{M}_{\delta(t)}\left(\mathbb{R}^{d}\right)}
\end{gathered}
$$

for $1 \leq q<\infty$.

(ii) For any $u_{0} \in L_{\varepsilon}^{\infty}\left(\mathbb{R}^{d}\right)$ we have

$$
\|u(t)\|_{L_{\delta}^{\infty}\left(\mathbb{R}^{d}\right)} \leq(1+4 \delta t)^{d}\left\|u_{0}\right\|_{L_{\delta(t)}^{\infty}\left(\mathbb{R}^{d}\right)} .
$$

(iii) For any $u_{0} \in L_{p \varepsilon}^{p}\left(\mathbb{R}^{d}\right)$ with $1 \leq p<\infty$ and for any $1 \leq p \leq q<\infty$ we have

$$
\begin{gathered}
\|u(t)\|_{L_{q \delta}^{q}\left(\mathbb{R}^{d}\right)} \leq c_{p, q} \frac{1}{(4 \delta t)^{\frac{d}{2}\left(\frac{1}{p}-\frac{1}{q}\right)}}(1+4 \delta t)^{\frac{d}{2}\left(1-\frac{1}{q}\right)}\left\|u_{0}\right\|_{L_{p \delta(t)}^{p}\left(\mathbb{R}^{d}\right)} \\
\|u(t)\|_{L_{\delta}^{\infty}\left(\mathbb{R}^{d}\right)} \leq c_{p, \infty} \frac{1}{(4 \pi t)^{\frac{d}{2 p}}}(1+4 \delta t)^{\frac{d}{2}}\left\|u_{0}\right\|_{L_{p \delta(t)}^{p}\left(\mathbb{R}^{d}\right)}
\end{gathered}
$$

which hold for $0<t<T(p \varepsilon)=\frac{1}{p} T(\varepsilon)$ if $\delta \geq p^{\prime} \varepsilon$ or for $0<t \leq T(\varepsilon)-T(\delta)$ if $\delta<p^{\prime} \varepsilon$.

Proof. Just notice that for $u_{0} \in \mathcal{M}_{\varepsilon}\left(\mathbb{R}^{d}\right)$, estimates (3.2), (4.5) and (4.9) remain valid as long as $\delta(t) \geq \varepsilon$ which leads to $0<t \leq T(\varepsilon)-T(\delta)$. Hence we get part (i). The same happens for $u_{0} \in L_{\varepsilon}^{\infty}\left(\mathbb{R}^{d}\right)$ in (4.6) and we get (ii).

Finally, for part (iii), for $u_{0} \in L_{p \varepsilon}^{p}\left(\mathbb{R}^{d}\right) \subset L_{p \varepsilon}^{1}\left(\mathbb{R}^{d}\right)$, (4.10) and (4.11) remain valid as long as $0<t<T(p \varepsilon)=\frac{1}{p} T(\varepsilon)$ and $\delta(t) \geq \varepsilon$, i. e. $0<t \leq T(\varepsilon)-T(\delta)$. Therefore, if $\delta \geq p^{\prime} \varepsilon$ the estimates are valid for all $0<t<T(p \varepsilon)=\frac{1}{p} T(\varepsilon)$. On the other hand, if $\delta<p^{\prime} \varepsilon$ the estimates are valid for all $0<t \leq T(\varepsilon)-T(\delta)$.

We now prove a dual version of this result, in which the solution is estimated in a norm that depends on time but the initial space is fixed.

Proposition 4.11. Assume that $\varepsilon>0$ and $0<t<T(\varepsilon)=\frac{1}{4 \varepsilon}$ and define

$$
\varepsilon(t):=\frac{1}{4(T(\varepsilon)-t)}=\frac{\varepsilon}{(1-4 \varepsilon t)} .
$$

(i) For any $u_{0} \in \mathcal{M}_{\varepsilon}\left(\mathbb{R}^{d}\right)$ we have

$$
\|u(t)\|_{L_{\varepsilon(t)}^{1}\left(\mathbb{R}^{d}\right)} \leq\left\|u_{0}\right\|_{\mathcal{M}_{\varepsilon}\left(\mathbb{R}^{d}\right)}
$$


with equality if $u_{0} \geq 0$, and also

$$
\begin{aligned}
\|u(t)\|_{L_{\varepsilon(t)}^{\infty}\left(\mathbb{R}^{d}\right)} & \leq \frac{1}{(4 \pi t)^{d / 2}} \frac{1}{(1-4 \varepsilon t)^{d / 2}}\left\|u_{0}\right\|_{\mathcal{M}_{\varepsilon}\left(\mathbb{R}^{d}\right)} \\
\|u(t)\|_{L_{q \varepsilon(t)}^{q}\left(\mathbb{R}^{d}\right)} & \leq \frac{q^{d / 2 q}}{\varepsilon^{\frac{d}{2}\left(1-\frac{1}{q}\right)}} \frac{1}{(4 t)^{\frac{d}{2}\left(1-\frac{1}{q}\right)}}\left\|u_{0}\right\|_{\mathcal{M}_{\varepsilon}\left(\mathbb{R}^{d}\right)}
\end{aligned}
$$

for $1 \leq q<\infty$.

(ii) For any $u_{0} \in L_{\varepsilon}^{\infty}\left(\mathbb{R}^{d}\right)$ we have

$$
\|u(t)\|_{L_{\varepsilon(t)}^{\infty}\left(\mathbb{R}^{d}\right)} \leq \frac{1}{(1-4 \varepsilon t)^{d}}\left\|u_{0}\right\|_{L_{\varepsilon}^{\infty}\left(\mathbb{R}^{d}\right)} .
$$

(iii) For any $u_{0} \in L_{p \varepsilon}^{p}\left(\mathbb{R}^{d}\right)$ with $1 \leq p<\infty$ and for any $1 \leq p \leq q<\infty$ we have

$$
\begin{aligned}
\|u(t)\|_{L_{q \varepsilon(t)}^{q}\left(\mathbb{R}^{d}\right)} & \leq \frac{c_{p, q}}{\varepsilon^{\frac{d}{2}\left(\frac{1}{p}-\frac{1}{q}\right)}} \frac{1}{(4 t)^{\frac{d}{2}\left(\frac{1}{p}-\frac{1}{q}\right)}} \frac{1}{(1-4 \varepsilon t)^{\frac{d}{2}\left(1-\frac{1}{p}\right)}}\left\|u_{0}\right\|_{L_{p \varepsilon}^{p}\left(\mathbb{R}^{d}\right)} \\
\|u(t)\|_{L_{\varepsilon(t)}^{\infty}\left(\mathbb{R}^{d}\right)} & \leq c_{p, \infty} \frac{1}{(4 \pi t)^{\frac{d}{2 p}}} \frac{1}{(1-4 \varepsilon t)^{\frac{d}{2}}}\left\|u_{0}\right\|_{L_{p \varepsilon}^{p}\left(\mathbb{R}^{d}\right)} .
\end{aligned}
$$

Proof. Since $\varepsilon>0$ and $0<t<T(\varepsilon)$, we set $\delta=\varepsilon(t)=\frac{1}{4(T(\varepsilon)-t)}=\frac{\varepsilon}{(1-4 \varepsilon t)}>\varepsilon$ and then $t=\frac{1}{4 \varepsilon}-\frac{1}{4 \delta}=T(\varepsilon)-T(\delta)$ and $\delta(t)=\frac{\delta}{1+4 \delta t}=\varepsilon$. Hence $1+4 \delta t=\frac{\varepsilon(t)}{\varepsilon}=\frac{1}{1-4 \varepsilon t}$ while $\frac{1+4 \delta t}{\delta}=\frac{1}{\varepsilon}$.

The result follows then from Proposition 4.10.

Notice that estimates on derivatives in (3.3) (4.15) and (4.16), can be extended along the same lines.

As a consequence of Propositions 4.10 and 4.11, if for $\varepsilon>0$ we define

$$
X_{\varepsilon}^{p}:= \begin{cases}L_{p \varepsilon}^{p}\left(\mathbb{R}^{d}\right), & \text { if } 1 \leq p<\infty \\ L_{\varepsilon}^{p}\left(\mathbb{R}^{d}\right), & \text { if } p=\infty .\end{cases}
$$

then for any $1 \leq p \leq q \leq \infty$ (even $t=0$ if $p=q$ ) the maps

$$
S(t): X_{\delta(t)}^{p} \longrightarrow X_{\delta}^{q}, \quad \delta(t)=\frac{\delta}{1+4 \delta t}
$$

and

$$
S(t): X_{\varepsilon}^{p}\left(\mathbb{R}^{d}\right) \longrightarrow X_{\varepsilon(t)}^{q}\left(\mathbb{R}^{d}\right), \quad \varepsilon(t)=\frac{1}{4(T(\varepsilon)-t)}=\frac{\varepsilon}{(1-4 \varepsilon t)},
$$

are continuous and Propositions 4.10 and 4.11 provide estimates on their norms. 


\section{$5 \quad$ Growth and decay as $t \rightarrow \infty$}

As a consequence of the results above we obtain the following characterization of the initial data for which the solution of the heat equation stays bounded or decays to zero in the Fréchet spaces $L_{0}^{q}\left(\mathbb{R}^{d}\right)$.

Proposition 5.1. (i) If $u_{0} \in \mathcal{M}_{0, B}\left(\mathbb{R}^{d}\right)$ as in Definition 3.6 then $u(t)=S(t) u_{0}$ is bounded in $L_{0}^{q}\left(\mathbb{R}^{d}\right), 1 \leq q \leq \infty$, for $t \geq 1$ while it decays to zero in $L_{0}^{q}\left(\mathbb{R}^{d}\right), 1 \leq q \leq \infty$, as $t \rightarrow \infty$ provided

$$
\left\|u_{0}\right\|_{\mathcal{M}_{\varepsilon}\left(\mathbb{R}^{d}\right)} \rightarrow 0, \quad \varepsilon \rightarrow 0
$$

a condition that is equivalent to $u(x, t) \rightarrow 0$ uniformly in sets $\frac{|x|}{\sqrt{t}} \leq R$.

The converse is also true if $u_{0} \geq 0$.

(ii) If $u_{0} \in L_{0}^{\infty}\left(\mathbb{R}^{d}\right)$ then $u(t)=S(t) u_{0}$ is bounded in $L_{0}^{\infty}\left(\mathbb{R}^{d}\right)$ if

$$
\frac{1}{\varepsilon^{d}}\left\|u_{0}\right\|_{L_{\varepsilon}^{\infty}\left(\mathbb{R}^{d}\right)} \leq C, \quad \varepsilon \rightarrow 0
$$

while it decays to zero in $L_{0}^{\infty}\left(\mathbb{R}^{d}\right)$ provided

$$
\frac{1}{\varepsilon^{d}}\left\|u_{0}\right\|_{L_{\varepsilon}^{\infty}\left(\mathbb{R}^{d}\right)} \rightarrow 0, \quad \varepsilon \rightarrow 0 .
$$

(iii) If $u_{0} \in L_{0}^{p}\left(\mathbb{R}^{d}\right), 1 \leq p<\infty$, then $u(t)=S(t) u_{0}$ is bounded in $L_{0}^{q}\left(\mathbb{R}^{d}\right), p \leq q \leq \infty$ if

$$
\frac{1}{\varepsilon^{\frac{d}{2}\left(1-\frac{1}{p}\right)}}\left\|u_{0}\right\|_{L_{\varepsilon}^{p}\left(\mathbb{R}^{d}\right)} \leq C, \quad \varepsilon \rightarrow 0
$$

while it decays to zero in $L_{0}^{q}\left(\mathbb{R}^{d}\right)$ provided

$$
\frac{1}{\varepsilon^{\frac{d}{2}\left(1-\frac{1}{p}\right)}}\left\|u_{0}\right\|_{L_{\varepsilon}^{p}\left(\mathbb{R}^{d}\right)} \rightarrow 0, \quad \varepsilon \rightarrow 0 .
$$

Proof. Part (i) for $q=1$ follows from (3.2) in Proposition 3.3 and Proposition 6.1 in [6]. For $1<q \leq \infty$ it follows from (4.5) and (4.9). For this note that in these estimates we take $\varepsilon=\delta(t)=\frac{\delta}{1+4 \delta t} \rightarrow 0$ as $t \rightarrow \infty$ and use that the factor $\left(\frac{1+4 \delta t}{4 \delta t}\right)^{\frac{d}{2}\left(1-\frac{1}{q}\right)}$ is bounded for $t>0$. The converse follows from Proposition 3.4 above, using that convergence in $L_{0}^{q}\left(\mathbb{R}^{d}\right)$ implies convergence in $L_{0}^{1}\left(\mathbb{R}^{d}\right)$.

Part (ii) follows from (4.6) while part (iii) follows from (4.10) and (4.11). For this note again that we take $\varepsilon=\delta(t)=\frac{\delta}{1+4 \delta t} \rightarrow 0$ as $t \rightarrow \infty$ and in (4.6) the factor $(1+4 \delta t)^{d} \approx \frac{1}{\varepsilon^{d}}$, while in (4.10) and (4.11) for large $t$ the factor $\frac{1}{(4 \delta t)^{\frac{d}{2}\left(\frac{1}{p}-\frac{1}{q}\right)}}(1+4 \delta t)^{\frac{d}{2}\left(1-\frac{1}{q}\right)} \approx t^{\frac{d}{2}\left(1-\frac{1}{p}\right)} \approx \varepsilon^{-\frac{d}{2}\left(1-\frac{1}{p}\right)}$.

Observe that an analogous result can be obtained for the derivatives $D^{\alpha} u(x, t)$, from (4.15), (4.17) by adding a factor $\varepsilon^{|\alpha| / 2}$ to the estimates on $u_{0}$ above.

Note that since we are dealing with initial data that can be very large as $|x| \rightarrow \infty$, equation (1.1) has some unusual properties. 
Lemma 5.2. The spectrum of $-\Delta$, as an operator on $L_{0}^{1}\left(\mathbb{R}^{d}\right)$, is the whole of $\mathbb{R}$.

Proof. For any $\mu>0$, take $\omega \in \mathbb{R}^{d}$ such that $|\omega|^{2}=\mu$. Then the function $\varphi(x)=\mathrm{e}^{\omega x} \in L_{0}^{1}\left(\mathbb{R}^{d}\right)$ satisfies

$$
-\Delta \varphi=-\mu \varphi, \quad \mu=|\omega|^{2} .
$$

On the other hand, $\phi(x)=\mathrm{e}^{i \omega x} \in L_{0}^{1}\left(\mathbb{R}^{d}\right)$ satisfies

$$
-\Delta \phi=\mu \phi, \quad \mu=|\omega|^{2} .
$$

This implies that for any $\mu>0$ and $\omega$ as above

$$
u(x, t)=\mathrm{e}^{\mu t+\omega x} \quad x \in \mathbb{R}^{d}, t>0
$$

is a globally defined solution of $(1.1)$ in $L_{0}^{1}\left(\mathbb{R}^{d}\right)$. In particular, for any $\delta>0$ the $L_{\delta}^{1}\left(\mathbb{R}^{d}\right)$ norm grows like $\mathrm{e}^{\mu t}=\mathrm{e}^{|\omega|^{2} t}$ :

$$
\|u(t)\|_{L_{\delta}^{1}\left(\mathbb{R}^{d}\right)}=\mathrm{e}^{|\omega|^{2} t}\left\|u_{0}\right\|_{\mathcal{M}_{\delta}\left(\mathbb{R}^{d}\right)}
$$

with $u_{0}(x)=\mathrm{e}^{\omega x}$. Hence the heat semigroup $S(t)$ has solutions that grow at an arbitrarily large exponential rate in the $L_{\delta}^{1}\left(\mathbb{R}^{d}\right)$ norm, for every fixed $\delta>0$.

Note however that estimate (3.2) reads

$$
\|u(t)\|_{L_{\delta}^{1}\left(\mathbb{R}^{d}\right)}=\mathrm{e}^{\mu t}\left\|u_{0}\right\|_{\mathcal{M}_{\delta}\left(\mathbb{R}^{d}\right)} \leq\left\|u_{0}\right\|_{\mathcal{M}_{\delta(t)}\left(\mathbb{R}^{d}\right)}
$$

with $\delta(t)=\frac{\delta}{1+4 \delta t}$, and a relatively elementary computation that involves completing the square in the exponential yields

$$
\left\|u_{0}\right\|_{\mathcal{M}_{\delta(t)}\left(\mathbb{R}^{d}\right)}=\mathrm{e}^{\frac{|\omega|^{2}}{4 \delta(t)}} \approx \mathrm{e}^{|\omega|^{2} t}
$$

for large $t$.

On the other hand, (3.3) reads

$$
\left\|D_{x}^{\alpha} u(t)\right\|_{L_{\delta}^{1}\left(\mathbb{R}^{d}\right)}=\left|\omega^{\alpha}\right| \mathrm{e}^{\mu t}\left\|u_{0}\right\|_{\mathcal{M}_{\delta}\left(\mathbb{R}^{d}\right)} \leq \frac{c_{\alpha, \gamma}}{t^{\frac{|\alpha|}{2}}}\left\|u_{0}\right\|_{\mathcal{M}_{\tilde{\delta}(t)}\left(\mathbb{R}^{d}\right)} .
$$

with $\tilde{\delta}(t)=\frac{\delta}{1+4 \gamma \delta t}$.

This gives a good indication why we cannot use a single $L_{\varepsilon}^{1}\left(\mathbb{R}^{d}\right)$ norm to study the dynamics of solution in $L_{0}^{1}\left(\mathbb{R}^{d}\right)$, but instead we have to use the Fréchet structure.

\section{Duality}

Our goal in this section is to prove that the heat solutions for large initial data in Propositions 3.3 and 4.8 are in natural duality with heat solutions for 'very rapidly' decaying initial data. This will give a suitable and natural integrability of heat solutions for large initial data against very rapidly decaying test functions, see Propositions 6.11 and 6.13 below. For this we first identify the dual space of $L_{\varepsilon}^{p}\left(\mathbb{R}^{d}\right)$. 


\subsection{Spaces of very rapidly decaying functions}

We define for $\varepsilon>0$ the weights, see (2.6),

$$
\rho_{\varepsilon}(x)=\left(\frac{\varepsilon}{\pi}\right)^{d / 2} \mathrm{e}^{-\varepsilon|x|^{2}}, \quad \rho_{-\varepsilon}(x)=\frac{1}{\rho_{\varepsilon}(x)}=\left(\frac{\pi}{\varepsilon}\right)^{d / 2} \mathrm{e}^{\varepsilon|x|^{2}}, \quad \rho_{0}(x)=1 .
$$

Clearly for suitable constants we have $\rho_{\varepsilon} \rho_{\delta}=c_{\varepsilon, \delta} \rho_{\varepsilon+\delta}$ and $\rho_{\varepsilon}^{\delta}=c_{\varepsilon, \delta} \rho_{\delta \varepsilon}$ for $\varepsilon, \delta \in \mathbb{R}$.

In an analogous way as in Sections 2 and 4 we can define for $\varepsilon>0$ the following Banach spaces:

$$
\mathcal{M}_{-\varepsilon}\left(\mathbb{R}^{d}\right):=\left\{\mu \in \mathcal{M}_{\mathrm{loc}}\left(\mathbb{R}^{d}\right): \int_{\mathbb{R}^{d}} \mathrm{e}^{\varepsilon|x|^{2}} \mathrm{~d}|\mu(x)|<\infty\right\} ;
$$

i.e. $\mathrm{e}^{\varepsilon|x|^{2}} \in L^{1}(d|\mu|)$, with the norm

$$
\|\mu\|_{\mathcal{M}_{-\varepsilon}\left(\mathbb{R}^{d}\right)}:=\int_{\mathbb{R}^{d}} \rho_{-\varepsilon}(x) \mathrm{d}|\mu(x)| .
$$

For $1 \leq p<\infty$

$$
L_{-\varepsilon}^{p}\left(\mathbb{R}^{d}\right):=\left\{f \in L_{\mathrm{loc}}^{p}\left(\mathbb{R}^{d}\right): \int_{\mathbb{R}^{d}} \rho_{-\varepsilon}(x)|f(x)|^{p} \mathrm{~d} x<\infty\right\}
$$

with the norm

$$
\|f\|_{L_{-\varepsilon}^{p}\left(\mathbb{R}^{d}\right)}:=\left(\int_{\mathbb{R}^{d}} \rho_{-\varepsilon}(x)|f(x)|^{p} \mathrm{~d} x\right)^{\frac{1}{p}}
$$

and for $p=\infty$

$$
L_{-\varepsilon}^{\infty}\left(\mathbb{R}^{d}\right):=\left\{f \in L_{\mathrm{loc}}^{\infty}\left(\mathbb{R}^{d}\right): \sup _{x \in \mathbb{R}^{d}} \rho_{-\varepsilon}(x)|f(x)|<\infty\right\}
$$

with the norm

$$
\|f\|_{L_{-\varepsilon}^{\infty}\left(\mathbb{R}^{d}\right)}:=\sup _{x \in \mathbb{R}^{d}} \rho_{-\varepsilon}(x)|f(x)| .
$$

Obviously $L_{-\varepsilon}^{1}\left(\mathbb{R}^{d}\right) \subset \mathcal{M}_{-\varepsilon}\left(\mathbb{R}^{d}\right)$ with equal norms.

Finally, we define the subspace of (6.3)

$$
C_{-\varepsilon, 0}\left(\mathbb{R}^{d}\right)=\left\{f \in L_{\mathrm{loc}}^{1}\left(\mathbb{R}^{d}\right): \rho_{-\varepsilon} f \in C_{0}\left(\mathbb{R}^{d}\right)\right\} \subset L_{-\varepsilon}^{\infty}\left(\mathbb{R}^{d}\right)
$$

with the norm (6.4). Observe that all these spaces are made of measures or functions that decay 'very rapidly' as $|x| \rightarrow \infty$, in some sense 'faster than $\mathrm{e}^{-\varepsilon|x|^{2}}$,

Before continuing, observe that, analogously to Lemma 4.1, we have the following results.

Lemma 6.1. (i) The spaces $\mathcal{M}_{-\varepsilon}\left(\mathbb{R}^{d}\right)$ and $L_{-\varepsilon}^{p}\left(\mathbb{R}^{d}\right), 1 \leq p \leq \infty$, are decreasing in $\varepsilon>0$.

(ii) For any $\varepsilon<\gamma$ and $1 \leq q<p \leq \infty$ we have

$$
L_{-\gamma}^{p}\left(\mathbb{R}^{d}\right) \subset L_{-\varepsilon}^{q}\left(\mathbb{R}^{d}\right)
$$


(iii) For $\varepsilon>0$ and $1 \leq p \leq \infty$

$$
L_{-\varepsilon}^{p}\left(\mathbb{R}^{d}\right) \subset L^{p}\left(\mathbb{R}^{d}\right) \cap L^{1}\left(\mathbb{R}^{d}\right)
$$

and

$$
\mathcal{M}_{-\varepsilon}\left(\mathbb{R}^{d}\right) \subset \mathcal{M}_{\mathrm{BTV}}\left(\mathbb{R}^{d}\right)
$$

the space of measures of bounded total variation.

Proof. (i) Analogously to (2.7) and (2.8) we have, for $0<\varepsilon_{1}<\varepsilon_{2}$,

$$
\rho_{-\varepsilon_{2}}(x) \geq\left(\frac{\varepsilon_{1}}{\varepsilon_{2}}\right)^{d / 2} \rho_{-\varepsilon_{1}}(x), \quad x \in \mathbb{R}^{d}
$$

which implies

$$
\|\mu\|_{\mathcal{M}_{-\varepsilon_{1}}\left(\mathbb{R}^{d}\right)} \leq\left(\frac{\varepsilon_{2}}{\varepsilon_{1}}\right)^{d / 2}\|\mu\|_{\mathcal{M}_{-\varepsilon_{2}}\left(\mathbb{R}^{d}\right)}, \quad\|f\|_{L_{-\varepsilon_{1}}^{p}\left(\mathbb{R}^{d}\right)} \leq\left(\frac{\varepsilon_{2}}{\varepsilon_{1}}\right)^{d / 2}\|f\|_{L_{-\varepsilon_{2}}^{p}\left(\mathbb{R}^{d}\right)} .
$$

Hence

$$
\mathcal{M}_{-\varepsilon_{2}}\left(\mathbb{R}^{d}\right) \subset \mathcal{M}_{-\varepsilon_{1}}\left(\mathbb{R}^{d}\right), \quad L_{-\varepsilon_{2}}^{p}\left(\mathbb{R}^{d}\right) \subset L_{-\varepsilon_{1}}^{p}\left(\mathbb{R}^{d}\right) .
$$

(ii) If $f \in L_{-\gamma}^{p}\left(\mathbb{R}^{d}\right)$, with $\gamma>0$ then if $p>q \geq 1$, then we have for a suitable $\alpha>1$, to be chosen later

$$
\int_{\mathbb{R}^{d}}|f(x)|^{q} \mathrm{e}^{\varepsilon|x|^{2}} \mathrm{~d} x=\int_{\mathbb{R}^{d}}|f(x)|^{q} \mathrm{e}^{\varepsilon \alpha|x|^{2}} \mathrm{e}^{-(\alpha-1) \varepsilon|x|^{2}} \mathrm{~d} x
$$

and Hölder's inequality gives

$$
\int_{\mathbb{R}^{d}}|f(x)|^{q} \mathrm{e}^{\varepsilon|x|^{2}} \mathrm{~d} x \leq\left[\int_{\mathbb{R}^{d}}|f(x)|^{p} \mathrm{e}^{\frac{\varepsilon \alpha p}{q}|x|^{2}} \mathrm{~d} x\right]^{\frac{q}{p}}\left[\int_{\mathbb{R}^{d}} \mathrm{e}^{\frac{-(\alpha-1) \varepsilon p}{p-q}|x|^{2}} \mathrm{~d} x\right]^{\frac{p-q}{p}} .
$$

Then if $\gamma>\varepsilon$ we chose $1<\alpha=\frac{q \gamma}{p \varepsilon}<\frac{\gamma}{\varepsilon}$ and then

$$
\int_{\mathbb{R}^{d}}|f(x)|{ }^{q} \mathrm{e}^{\varepsilon|x|^{2}} \mathrm{~d} x \leq c_{p, q, \gamma, \varepsilon}\left[\int_{\mathbb{R}^{d}}|f(x)|^{p} \mathrm{e}^{\gamma|x|^{2}} \mathrm{~d} x\right]^{\frac{q}{p}} .
$$

Analogously if $f \in L_{-\gamma}^{\infty}\left(\mathbb{R}^{d}\right)$, with $\gamma>0$

$$
\int_{\mathbb{R}^{d}}|f(x)|^{q} \mathrm{e}^{\varepsilon|x|^{2}} \mathrm{~d} x=\int_{\mathbb{R}^{d}}|f(x)|^{q} \mathrm{e}^{q \gamma|x|^{2}} \mathrm{e}^{-(q \gamma-\varepsilon)|x|^{2}} \mathrm{~d} x \leq\|f\|_{L_{\gamma}^{\infty}\left(\mathbb{R}^{d}\right)}^{q} \int_{\mathbb{R}^{d}} \mathrm{e}^{-(q \gamma-\varepsilon)|x|^{2}} \mathrm{~d} x
$$

which is finite provided $q \geq 1>\frac{\varepsilon}{\gamma}$.

For (iii) just observe that for $f \in L_{-\varepsilon}^{p}\left(\mathbb{R}^{d}\right)$

$$
\int_{\mathbb{R}^{d}}|f|^{p}(x) \mathrm{d} x \leq \int_{\mathbb{R}^{d}} \mathrm{e}^{\varepsilon|x|^{2}}|f|^{p}(x) \mathrm{d} x \quad \int_{\mathbb{R}^{d}}|f|(x) \mathrm{d} x \leq \int_{\mathbb{R}^{d}} \mathrm{e}^{\frac{\varepsilon}{p}|x|^{2}}|f|(x) \mathrm{e}^{\frac{-\varepsilon}{p}|x|^{2}} \mathrm{~d} x
$$

and use Hölder's inequality. The case $p=\infty$ is similar. Finally for $\mu \in \mathcal{M}_{-\varepsilon}\left(\mathbb{R}^{d}\right)$

$$
\int_{\mathbb{R}^{d}} \mathrm{~d}|\mu|(x) \leq \int_{\mathbb{R}^{d}} \mathrm{e}^{\varepsilon|x|^{2}} \mathrm{~d}|\mu|(x) .
$$


As in Lemma 4.2 and Remark 4.3 the operator $\Phi_{-\varepsilon}(f):=\rho_{-\varepsilon} f$ is an isometric isomorphism between the following pairs of spaces:

$$
\begin{aligned}
& \left.\Phi_{-\varepsilon}: \mathcal{M}_{-\varepsilon}\left(\mathbb{R}^{d}\right) \rightarrow \mathcal{M}_{\mathrm{BTV}}\left(\mathbb{R}^{d}\right) \text { (in the sense that } \mathrm{d} \Phi_{-\varepsilon}(\mu)=\rho_{-\varepsilon} \mathrm{d} \mu\right), \\
& \Phi_{-\varepsilon}: L_{-\varepsilon}^{1}\left(\mathbb{R}^{d}\right) \rightarrow L^{1}\left(\mathbb{R}^{d}\right), \\
& \Phi_{-\varepsilon}: L_{-p \varepsilon}^{p}\left(\mathbb{R}^{d}\right) \rightarrow L^{p}\left(\mathbb{R}^{d}\right), \\
& \Phi_{-\varepsilon}: L_{-\varepsilon}^{\infty}\left(\mathbb{R}^{d}\right) \rightarrow L^{\infty}\left(\mathbb{R}^{d}\right), \text { and } \\
& \Phi_{-\varepsilon}: C_{-\varepsilon, 0}\left(\mathbb{R}^{d}\right) \rightarrow C_{0}\left(\mathbb{R}^{d}\right) .
\end{aligned}
$$

Clearly $\Phi_{\varepsilon}$ and $\Phi_{-\varepsilon}$ are the inverse of each other.

These spaces and their relationships are summarized in the following diagram.

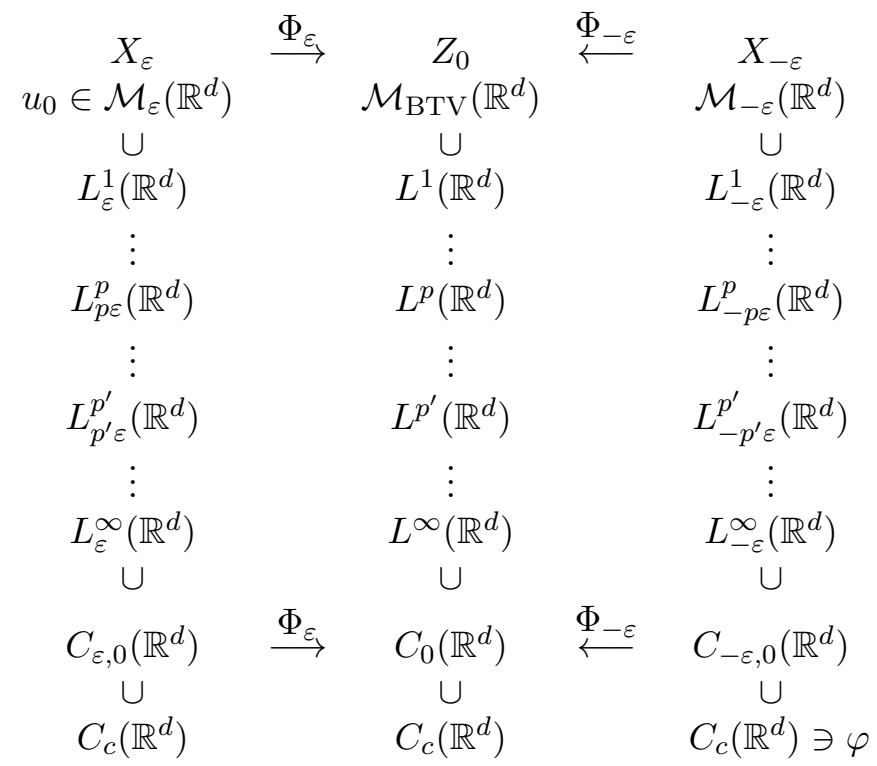

Observe that analogously to Lemma 4.2 the spaces $L_{-p \varepsilon}^{p}\left(\mathbb{R}^{d}\right)$ are the complex interpolation spaces between $L_{-\varepsilon}^{1}\left(\mathbb{R}^{d}\right)$ and $L_{-\varepsilon}^{\infty}\left(\mathbb{R}^{d}\right)$ and are given by

$$
\left[L_{-\varepsilon}^{1}\left(\mathbb{R}^{d}\right), L_{-\varepsilon}^{\infty}\left(\mathbb{R}^{d}\right)\right]_{\frac{1}{p^{\prime}}}=\left\{f \in L_{\mathrm{loc}}^{1}\left(\mathbb{R}^{d}\right), \int_{\mathbb{R}^{d}} \rho_{-\varepsilon}^{p}(x)|f|^{p}(x) \mathrm{d} x<\infty\right\}=L_{-p \varepsilon}^{p}\left(\mathbb{R}^{d}\right)
$$

with norms

$$
\|f\|_{p,-\varepsilon}:=\left\|f \rho_{-\varepsilon}\right\|_{L^{p}\left(\mathbb{R}^{d}\right)}=\left(\frac{\pi}{\varepsilon}\right)^{\frac{d}{2}\left(1-\frac{1}{p}\right)} p^{\frac{d}{2 p}}\|f\|_{L_{-p \varepsilon}^{p}\left(\mathbb{R}^{d}\right)}
$$

since $\rho_{-\varepsilon}^{p}(x)=c_{\varepsilon, p} \rho_{-p \varepsilon}(x)$, with $c_{\varepsilon, p}=\left(\frac{\pi}{\varepsilon}\right)^{\frac{d}{2}(p-1)} p^{\frac{d}{2}}$.

We now observe the following density results.

Lemma 6.2. For any $\varepsilon \neq 0$, the set $C_{c}\left(\mathbb{R}^{d}\right)$ is dense in $L_{\varepsilon}^{p}\left(\mathbb{R}^{d}\right)$, for $1 \leq p<\infty$, and in $C_{\varepsilon, 0}\left(\mathbb{R}^{d}\right)$.

Proof. Observe that $f \in X$ with $X=L_{\varepsilon}^{p}\left(\mathbb{R}^{d}\right)$ or $X=C_{\varepsilon, 0}\left(\mathbb{R}^{d}\right)$ if and only if $\rho_{\varepsilon} f \in Y$ with $Y=L^{p}\left(\mathbb{R}^{d}\right)$ or $Y=C_{0}\left(\mathbb{R}^{d}\right)$. Then there exist $\varphi_{n} \in C_{c}\left(\mathbb{R}^{d}\right)$ such that $\varphi_{n} \rightarrow \rho_{\varepsilon} f$ in $Y$. Therefore $\rho_{-\varepsilon} \varphi_{n} \rightarrow f$ in $X$ and $\rho_{-\varepsilon} \varphi_{n} \in C_{c}\left(\mathbb{R}^{d}\right)$. 
Lemma 6.3. For $\varepsilon \neq 0, C_{\varepsilon, 0}\left(\mathbb{R}^{d}\right)$ is dense in $\mathcal{M}_{\varepsilon}\left(\mathbb{R}^{d}\right)$ in the sense of measures.

Proof. If $\mu \in \mathcal{M}_{\varepsilon}\left(\mathbb{R}^{d}\right)$ then $\rho_{\varepsilon} \mu \in \mathcal{M}_{\mathrm{BTV}}\left(\mathbb{R}^{d}\right)$ and then we claim that there exist $\varphi_{n} \in C_{0}\left(\mathbb{R}^{d}\right)$ such that $\varphi_{n} \rightarrow \rho_{\varepsilon} \mu$ in the sense of measures, that is, for every $\phi \in C_{c}\left(\mathbb{R}^{d}\right)$

$$
\int_{\mathbb{R}^{d}} \phi \varphi_{n} \rightarrow \int_{\mathbb{R}^{d}} \phi \rho_{\varepsilon} \mathrm{d} \mu
$$

Hence, taking $\eta=\phi \rho_{\varepsilon} \in C_{c}\left(\mathbb{R}^{d}\right)$, we get

$$
\int_{\mathbb{R}^{d}} \eta \rho_{-\varepsilon} \varphi_{n} \rightarrow \int_{\mathbb{R}^{d}} \eta \mathrm{d} \mu
$$

for every $\eta \in C_{c}\left(\mathbb{R}^{d}\right)$. That is $\rho_{-\varepsilon} \varphi_{n} \rightarrow \mu$ in the sense of measures and $\rho_{-\varepsilon} \varphi_{n} \in C_{\varepsilon, 0}\left(\mathbb{R}^{d}\right)$.

To prove the claim above note that from Lemma 5.3 in $[6]$ we have that for $\mu \in \mathcal{M}_{\mathrm{BTV}}\left(\mathbb{R}^{d}\right)$ the solution of the heat equation given by (2.2) satisfies

$$
\|S(t) \mu\|_{\mathrm{BTV}} \leq\|\mu\|_{\mathrm{BTV}}, \quad t>0
$$

and for every $1 \leq q \leq \infty$

$$
\|S(t) \mu\|_{L^{q}\left(\mathbb{R}^{d}\right)} \leq(4 \pi t)^{-\frac{d}{2}\left(1-\frac{1}{q}\right)}\|\mu\|_{\mathrm{BTV}}, \quad t>0 .
$$

and we also know that $S(t) \mu \rightarrow \mu$ in the sense of measures. Then it is enough to show that $S(t) \mu \in C_{0}\left(\mathbb{R}^{d}\right)$ as it is in $B U C\left(\mathbb{R}^{d}\right) \cap L^{1}\left(\mathbb{R}^{d}\right)$. Indeed if $f \in B U C\left(\mathbb{R}^{d}\right) \cap L^{1}\left(\mathbb{R}^{d}\right)$ and there exists $\left\{x_{n}\right\}_{n}$ with $\left|x_{n}\right| \rightarrow \infty$ and $L>0$ such that $\left|f\left(x_{n}\right)\right|>L$, then we can assume $\left|x_{n}-x_{m}\right| \geq \delta>0$ for $n \neq m$ and some $\delta>0$ and then since $f \in B U C\left(\mathbb{R}^{d}\right)$ we get some $r>0$ with $r<\delta$ and such that for $x \in B\left(x_{n}, r\right),|f(x)|>\frac{L}{2}$. But then $\int_{\mathbb{R}^{d}}|f|=\infty$ which is a contradiction.

Using this we can describe the dual spaces (and corresponding norms) of the spaces above.

Proposition 6.4. With the notation above we have, for $\varepsilon \neq 0$,

(i) $\left(L_{\varepsilon}^{1}\left(\mathbb{R}^{d}\right)\right)^{\prime}=L_{-\varepsilon}^{\infty}\left(\mathbb{R}^{d}\right)$, and $(f, g) \mapsto \int_{\mathbb{R}^{d}} f(x) g(x) \mathrm{d} x$ defines a duality pairing between these spaces such that

$$
\left|\int_{\mathbb{R}^{d}} f(x) g(x) \mathrm{d} x\right| \leq\|f\|_{L_{\varepsilon}^{1}\left(\mathbb{R}^{d}\right)}\|g\|_{L_{-\varepsilon}^{\infty}\left(\mathbb{R}^{d}\right)} .
$$

(ii) For $1<p<\infty$ and $\frac{1}{p}+\frac{1}{p^{\prime}}=1$,

$$
\left(L_{p \varepsilon}^{p}\left(\mathbb{R}^{d}\right)\right)^{\prime}=L_{-p^{\prime} \varepsilon}^{p^{\prime}}\left(\mathbb{R}^{d}\right),
$$

and $(f, g) \mapsto \int_{\mathbb{R}^{d}} f(x) g(x) \mathrm{d} x$ defines a duality pairing between these spaces such that

$$
\left|\int_{\mathbb{R}^{d}} f(x) g(x) \mathrm{d} x\right| \leq\|f\|\left\|_{p, \varepsilon}\right\| g\left\|_{p^{\prime},-\varepsilon}=\left(\frac{\varepsilon}{\pi}\right)^{\frac{d}{2}\left(\frac{1}{p^{\prime}}-\frac{1}{p}\right)}\left(\frac{p^{\prime p^{\prime}}}{p^{p}}\right)^{d / 2}\right\| f\left\|_{L_{p \varepsilon}^{p}\left(\mathbb{R}^{d}\right)}\right\| g \|_{L_{-p^{\prime} \varepsilon}^{p^{\prime}}\left(\mathbb{R}^{d}\right)} .
$$


(iii) $\mathcal{M}_{\varepsilon}\left(\mathbb{R}^{d}\right)=\left(C_{-\varepsilon, 0}\left(\mathbb{R}^{d}\right)\right)^{\prime}$ and $(f, \mu) \mapsto \int_{\mathbb{R}^{d}} f(x) \mathrm{d} \mu(x)$ defines a duality pairing between these spaces such that

$$
\left|\int_{\mathbb{R}^{d}} f(x) \mathrm{d} \mu(x)\right| \leq\|f\|_{L_{-\varepsilon}^{\infty}\left(\mathbb{R}^{d}\right)}\|\mu\|_{\mathcal{M}_{\varepsilon}\left(\mathbb{R}^{d}\right)}
$$

Proof. Observe that $L \in\left(L_{p \varepsilon}^{p}\left(\mathbb{R}^{d}\right)\right)^{\prime}$ for $1 \leq p<\infty$ if and only if $L \circ \Phi_{-\varepsilon} \in\left(L^{p}\left(\mathbb{R}^{d}\right)\right)^{\prime}$. Hence, there exists $h \in L^{p^{\prime}}\left(\mathbb{R}^{d}\right)$ such that for any $f \in L_{p \varepsilon}^{p}\left(\mathbb{R}^{d}\right)$

$$
L(f)=\int_{\mathbb{R}^{d}} f \rho_{\varepsilon} h .
$$

Hence, denoting $g=\rho_{\varepsilon} h \in L_{-p^{\prime} \varepsilon}^{p^{\prime}}\left(\mathbb{R}^{d}\right)$ if $1<p<\infty$ or $g \in L_{-\varepsilon}^{\infty}\left(\mathbb{R}^{d}\right)$ if $p=1$ we get the result. Notice also

$$
|L(f)| \leq\left\|f \rho_{\varepsilon}\right\|_{L^{p}\left(\mathbb{R}^{d}\right)}\|h\|_{L^{p^{\prime}\left(\mathbb{R}^{d}\right)}}=\|f\|_{p, \varepsilon}\|g\|_{p^{\prime},-\varepsilon}
$$

and the rest follows from (4.4) and (6.6).

Analogously $L \in\left(C_{-\varepsilon, 0}\left(\mathbb{R}^{d}\right)\right)^{\prime}$ if and only if $L \circ \Phi_{\varepsilon} \in\left(C_{0}\left(\mathbb{R}^{d}\right)\right)^{\prime}$. Hence, there exists a $\sigma \in \mathcal{M}_{\mathrm{BTV}}\left(\mathbb{R}^{d}\right)$ such that for any $f \in C_{-\varepsilon, 0}\left(\mathbb{R}^{d}\right)$

$$
L(f)=\int_{\mathbb{R}^{d}} f \rho_{-\varepsilon} \mathrm{d} \sigma=\int_{\mathbb{R}^{d}} f \mathrm{~d} \mu
$$

with $\mathrm{d} \mu=\rho_{-\varepsilon} \mathrm{d} \sigma \in \mathcal{M}_{\varepsilon}\left(\mathbb{R}^{d}\right)$. Also

$$
|L(f)| \leq\left\|f \rho_{-\varepsilon}\right\|_{C_{0}\left(\mathbb{R}^{d}\right)}\|\sigma\|_{\mathcal{M}_{\mathrm{BTV}}\left(\mathbb{R}^{d}\right)}=\|f\|_{L_{-\varepsilon}^{\infty}\left(\mathbb{R}^{d}\right)}\|\mu\|_{\mathcal{M}_{\varepsilon}\left(\mathbb{R}^{d}\right)} .
$$

Now for $1 \leq p \leq \infty$ we define

$$
L_{-0}^{p}\left(\mathbb{R}^{d}\right)=\bigcup_{\varepsilon>0} L_{-\varepsilon}^{p}\left(\mathbb{R}^{d}\right)
$$

using the definition we obtain the following characterization of the dual space of $L_{0}^{p}\left(\mathbb{R}^{d}\right)$.

Corollary 6.5. For $1 \leq p<\infty$

$$
\left(L_{0}^{p}\left(\mathbb{R}^{d}\right)\right)^{\prime}=L_{-0}^{p^{\prime}}\left(\mathbb{R}^{d}\right)
$$

Proof. Notice that $L \in\left(L_{0}^{p}\left(\mathbb{R}^{d}\right)\right)^{\prime}$ if and only if for some $\varepsilon>0, c_{\varepsilon}>0$ and for all $f \in L_{0}^{p}\left(\mathbb{R}^{d}\right)$ we have

$$
|L(f)| \leq c_{\varepsilon}\|f\|_{L_{\varepsilon}^{p}\left(\mathbb{R}^{d}\right)} .
$$

By Lemma 6.2 and density, the inequality above holds for any $f \in L_{\varepsilon}^{p}\left(\mathbb{R}^{d}\right)$. Thus $L \in\left(L_{\varepsilon}^{p}\left(\mathbb{R}^{d}\right)\right)^{\prime}$.

Conversely if $L \in \bigcup_{\varepsilon>0} L_{-\varepsilon}^{p^{\prime}}\left(\mathbb{R}^{d}\right)$ then for some $\varepsilon>0,|L(f)| \leq c_{\varepsilon}\|f\|_{L_{\varepsilon}^{p}\left(\mathbb{R}^{d}\right)}$ for all $f \in L_{\varepsilon}^{p}\left(\mathbb{R}^{d}\right)$ and thus for all $f \in L_{0}^{p}\left(\mathbb{R}^{d}\right)$. Hence $L \in\left(L_{0}^{p}\left(\mathbb{R}^{d}\right)\right)^{\prime}$. 


\subsection{The heat flow in spaces of very-rapidly decaying functions}

Now we analyze the heat semigroup (2.2) in the spaces of very rapidly decaying functions (6.1), (6.2), (6.3). Notice that from Lemma 6.1 all solutions with such initial data are globally defined. Also, like the estimates in Section 2, the estimates below imply that the solution enters, at each time, a larger space, as $\gamma(t)$ decreases with time. Even in this setting the solution 'loses integrability' as time increases.

Proposition 6.6. For $\gamma, t>0$ define $\gamma(t):=\frac{\gamma}{1+4 \gamma t}$.

(i) If $\varphi \in L_{-\gamma}^{\infty}\left(\mathbb{R}^{d}\right)$, that is $|\varphi(x)| \leq A \mathrm{e}^{-\gamma|x|^{2}}, x \in \mathbb{R}^{d}$, with $A=\left(\frac{\gamma}{\pi}\right)^{d / 2}\|\varphi\|_{L_{-\gamma}^{\infty}\left(\mathbb{R}^{d}\right)}$, then $u(t)=S(t) \varphi$ satisfies

$$
|u(x, t)| \leq \frac{A}{(1+4 \gamma t)^{d / 2}} \mathrm{e}^{-\frac{\gamma}{1+4 \gamma t}|x|^{2}}, \quad x \in \mathbb{R}^{d}, \quad t>0
$$

and so

$$
\|S(t) \varphi\|_{L_{-\gamma(t)}^{\infty}\left(\mathbb{R}^{d}\right)} \leq\|\varphi\|_{L_{-\gamma}^{\infty}\left(\mathbb{R}^{d}\right)} .
$$

(ii) If $\varphi \in \mathcal{M}_{-\gamma}\left(\mathbb{R}^{d}\right)$ then $u(t)=S(t) \varphi$ satisfies

$$
|u(x, t)| \leq \frac{1}{(4 \pi t)^{d / 2}} \mathrm{e}^{-\frac{\gamma}{1+4 \gamma t}|x|^{2}}\left(\frac{\gamma}{\pi}\right)^{d / 2}\|\varphi\|_{\mathcal{M}_{-\gamma}\left(\mathbb{R}^{d}\right)}, \quad x \in \mathbb{R}^{d}, \quad t>0
$$

and so

$$
\|S(t) \varphi\|_{L_{-\gamma(t)}^{\infty}\left(\mathbb{R}^{d}\right)} \leq\left(\frac{1+4 \gamma t}{4 \pi t}\right)^{d / 2}\|\varphi\|_{\mathcal{M}_{-\gamma}\left(\mathbb{R}^{d}\right)}
$$

(iii) If $\varphi \in \mathcal{M}_{-\gamma}\left(\mathbb{R}^{d}\right)$ then $u(t)=S(t) \varphi$ satisfies

$$
\|S(t) \varphi\|_{L_{-\gamma(t)}^{1}\left(\mathbb{R}^{d}\right)} \leq(1+4 \gamma t)^{d}\|\varphi\|_{\mathcal{M}_{-\gamma}\left(\mathbb{R}^{d}\right)}
$$

with equality if $\varphi \geq 0$.

Proof. Observe that

$$
|u(x, t)| \leq \frac{1}{(4 \pi t)^{d / 2}} \int_{\mathbb{R}^{d}} \mathrm{e}^{-|x-y|^{2} / 4 t} \mathrm{e}^{-\gamma|y|^{2}} \mathrm{e}^{\gamma|y|^{2}} \mathrm{~d}|\varphi(y)|
$$

so, completing the square yields

$$
\frac{|x-y|^{2}}{4 t}+\gamma|y|^{2}=\frac{1+4 \gamma t}{4 t}\left|y-\frac{1}{1+4 \gamma t} x\right|^{2}+\frac{\gamma|x|^{2}}{1+4 \gamma t}
$$

and then

$$
\mathrm{e}^{\frac{\gamma|x|^{2}}{1+4 \gamma t}}|u(x, t)| \leq \frac{1}{(4 \pi t)^{d / 2}} \int_{\mathbb{R}^{d}} \mathrm{e}^{-\frac{1+4 \gamma t}{4 t}\left|y-\frac{1}{1+4 \gamma t} x\right|^{2}} \mathrm{e}^{\gamma|y|^{2}} \mathrm{~d}|\varphi(y)| .
$$


Then for part (i) from (6.11) with $\varphi \in L_{-\gamma}^{\infty}\left(\mathbb{R}^{d}\right)$

$$
\begin{aligned}
\mathrm{e}^{\frac{\gamma|x|^{2}}{1+4 \gamma t}}|u(x, t)| & \leq \frac{A}{(4 \pi t)^{d / 2}} \int_{\mathbb{R}^{d}} \mathrm{e}^{-\frac{1+4 \gamma t}{4 t}\left|y-\frac{1}{1+4 \gamma t} x\right|^{2}} \mathrm{~d} y \\
& =\frac{A}{(4 \pi t)^{d / 2}} \int_{\mathbb{R}^{d}} \mathrm{e}^{-\frac{1+4 \gamma t}{4 t}|y|^{2}} \mathrm{~d} y \\
& =\frac{1}{(1+4 \gamma t)^{\frac{d}{2}}}\left(\frac{\gamma}{\pi}\right)^{d / 2}\|\varphi\|_{L_{-\gamma}^{\infty}\left(\mathbb{R}^{d}\right)}
\end{aligned}
$$

and the estimate follows.

For part (ii) from (6.11) we have

$$
\sup _{x \in \mathbb{R}^{d}} \mathrm{e}^{\frac{\gamma|x|^{2}}{1+4 \gamma t}}|u(x, t)| \leq \frac{1}{(4 \pi t)^{d / 2}} \int_{\mathbb{R}^{d}} \mathrm{e}^{\gamma|y|^{2}} \mathrm{~d}|\varphi(y)|=\frac{1}{(4 \pi t)^{d / 2}}\left(\frac{\gamma}{\pi}\right)^{d / 2}\|\varphi\|_{\mathcal{M}_{-\gamma}\left(\mathbb{R}^{d}\right)},
$$

and multiplying both sides by $\left(\frac{\pi}{\gamma}\right)^{d / 2}(1+4 \gamma t)^{\frac{d}{2}}$ the result follows.

Finally, for part (iii) from (6.11) we have

$$
\int_{\mathbb{R}^{d}} \mathrm{e}^{\frac{\gamma|x|^{2}}{1+4 \gamma t}}|u(x, t)| \mathrm{d} x \leq \frac{1}{(4 \pi t)^{d / 2}} \int_{\mathbb{R}^{d}} \int_{\mathbb{R}^{d}} \mathrm{e}^{-\frac{1+4 \gamma t}{4 t}\left|y-\frac{1}{1+4 \gamma t} x\right|^{2}} \mathrm{e}^{\gamma|y|^{2}} \mathrm{~d}|\varphi(y)| \mathrm{d} x
$$

and using Fubini's Theorem we obtain

$$
\begin{aligned}
\int_{\mathbb{R}^{d}} \mathrm{e}^{\frac{\gamma|x|^{2}}{1+4 \gamma t}}|u(x, t)| \mathrm{d} x & \leq \frac{1}{(4 \pi t)^{d / 2}} \int_{\mathbb{R}^{d}}\left(\int_{\mathbb{R}^{d}} \mathrm{e}^{-\frac{1+4 \gamma t}{4 t}\left|y-\frac{1}{1+4 \gamma t} x\right|^{2}} \mathrm{~d} x\right) \mathrm{e}^{\gamma|y|^{2}} \mathrm{~d}|\varphi(y)| \\
& =\frac{1}{(4 \pi t)^{d / 2}}\left(\int_{\mathbb{R}^{d}} \mathrm{e}^{\frac{-|x|^{2}}{4 t(1+4 \gamma t)}} \mathrm{d} x\right)\left(\int_{\mathbb{R}^{d}} \mathrm{e}^{\gamma|y|^{2}} \mathrm{~d}|\varphi(y)|\right) \\
& =(1+4 \gamma t)^{d / 2} \int_{\mathbb{R}^{d}} \mathrm{e}^{\gamma|y|^{2}} \mathrm{~d}|\varphi(y)|
\end{aligned}
$$

and multiplying both sides by $\left(\frac{\pi}{\gamma}\right)^{d / 2}(1+4 \gamma t)^{\frac{d}{2}}$ the result follows.

Clearly, all inequalities above become equal signs if $\varphi \geq 0$.

As a consequence we get the following estimates on the heat solutions for very rapidly decaying initial data.

Corollary 6.7. For $\gamma, t>0$ define $\gamma(t):=\frac{\gamma}{1+4 \gamma t}$.

(i) If $1 \leq p<\infty$ and $\varphi \in \mathcal{M}_{-\gamma}\left(\mathbb{R}^{d}\right)$

$$
\|S(t) \varphi\|_{L_{-p \gamma(t)}^{p}\left(\mathbb{R}^{d}\right)} \leq\left(\frac{\gamma}{\pi}\right)^{\frac{d}{2}\left(1-\frac{1}{p}\right)} \frac{1}{p^{d / 2 p}} \frac{(1+4 \gamma t)^{\frac{d}{p}}}{(4 \pi t)^{\frac{d}{2}\left(1-\frac{1}{p}\right)}}\|\varphi\|_{\mathcal{M}_{-\gamma}\left(\mathbb{R}^{d}\right)} .
$$


(ii) For $1 \leq p<\infty$ and $\varphi \in L_{-p \gamma}^{p}\left(\mathbb{R}^{d}\right)$

$$
\|S(t) \varphi\|_{L_{-\gamma(t)}^{\infty}\left(\mathbb{R}^{d}\right)} \leq\left(\frac{\pi}{\gamma}\right)^{\frac{d}{2}\left(1-\frac{1}{p}\right)} p^{d / 2 p}\left(\frac{1+4 \gamma t}{4 \pi t}\right)^{\frac{d}{2 p}}\|\varphi\|_{L_{-p \gamma}^{p}\left(\mathbb{R}^{d}\right)} .
$$

(iii) For $1 \leq p \leq q<\infty$ and $\varphi \in L_{-p \gamma}^{p}\left(\mathbb{R}^{d}\right)$

$$
\|S(t) \varphi\|_{L_{-q \gamma(t)}^{q}\left(\mathbb{R}^{d}\right)} \leq c_{p, q, \gamma} \frac{(1+4 \gamma t)^{\frac{d}{2}\left(\frac{1}{p}+\frac{2}{q}-1\right)}}{(4 \pi t)^{\frac{d}{2}\left(\frac{1}{p}-\frac{1}{q}\right)}}\|\varphi\|_{L_{-p \gamma}^{p}\left(\mathbb{R}^{d}\right)}
$$

$$
\text { where } c_{p, q, \gamma}=\left(\frac{\gamma}{\pi}\right)^{\frac{d}{2}\left(\frac{1}{p}-\frac{1}{q}\right)} \frac{p^{d / 2 p}}{q^{d / 2 q}} \text {. }
$$

Proof. (i) Interpolate (6.8) and (6.9) with $\theta=\frac{1}{p^{\prime}}$ to get

$$
\|S(t) \varphi\|_{p,-\gamma(t)} \leq(1+4 \gamma t)^{d(1-\theta)}\left(\frac{1+4 \gamma t}{4 \pi t}\right)^{\frac{d \theta}{2}}\|\varphi\|_{\mathcal{M}_{-\gamma}\left(\mathbb{R}^{d}\right)}=\frac{(1+4 \gamma t)^{\frac{d}{2}\left(1+\frac{1}{p}\right)}}{(4 \pi t)^{\frac{d}{2}\left(1-\frac{1}{p}\right)}}\|\varphi\|_{\mathcal{M}_{-\gamma}\left(\mathbb{R}^{d}\right)} .
$$

Then (6.6) gives

$$
\left(\frac{\pi}{\gamma(t)}\right)^{\frac{d}{2}\left(1-\frac{1}{p}\right)} p^{d / 2 p}\|S(t) \varphi\|_{L_{-p \gamma(t)}^{p}\left(\mathbb{R}^{d}\right)} \leq \frac{(1+4 \gamma t)^{\frac{d}{2}\left(1+\frac{1}{p}\right)}}{(4 \pi t)^{\frac{d}{2}\left(1-\frac{1}{p}\right)}}\|\varphi\|_{\mathcal{M}_{-\gamma}\left(\mathbb{R}^{d}\right)}
$$

and we get (6.12).

(ii) Interpolate (6.7), (6.8) for $\varphi \in L_{-\gamma}^{1}\left(\mathbb{R}^{d}\right)$ with $\theta=\frac{1}{p^{\prime}}$ to get

$$
\|S(t) \varphi\|_{L_{-\gamma(t)}^{\infty}\left(\mathbb{R}^{d}\right)} \leq\left(\frac{1+4 \gamma t}{4 \pi t}\right)^{\frac{d}{2}(1-\theta)}\|\varphi\|_{p,-\gamma}=\left(\frac{1+4 \gamma t}{4 \pi t}\right)^{\frac{d}{2 p}}\|\varphi\|_{p,-\gamma} .
$$

Then (6.6) gives

$$
\|S(t) \varphi\|_{L_{-\gamma(t)}^{\infty}\left(\mathbb{R}^{d}\right)} \leq\left(\frac{1+4 \gamma t}{4 \pi t}\right)^{\frac{d}{2 p}}\left(\frac{\pi}{\gamma}\right)^{\frac{d}{2}\left(1-\frac{1}{p}\right)} p^{d / 2 p}\|\varphi\|_{L_{-p \gamma}^{p}\left(\mathbb{R}^{d}\right)}
$$

and we get (6.13).

(iii) Interpolate (6.7) and (6.9) for $\varphi \in L_{-\gamma}^{1}\left(\mathbb{R}^{d}\right)$ with $\theta=\frac{1}{p^{\prime}}$ to obtain

$$
\|S(t) \varphi\|_{p,-\gamma(t)} \leq(1+4 \gamma t)^{d(1-\theta)}\|\varphi\|_{p,-\gamma}=(1+4 \gamma t)^{d / p}\|\varphi\|_{p,-\gamma} .
$$

Now interpolate (6.17) and (6.16) with $\theta=1-\frac{p}{q}$ to get, by reiteration of the interpolation,

$$
\|S(t) \varphi\|_{\left[L_{-p \gamma}^{p}\left(\mathbb{R}^{d}\right), L_{-\gamma}^{\infty}\left(\mathbb{R}^{d}\right)\right]_{\theta}}=\|S(t) \varphi\|_{q,-\gamma(t)}
$$

and then

$$
\|S(t) \varphi\|_{q,-\gamma(t)} \leq(1+4 \gamma t)^{\frac{d}{p}(1-\theta)}\left(\frac{1+4 \gamma t}{4 \pi t}\right)^{\frac{d \theta}{2 p}}\|\varphi\|_{p,-\gamma}=\frac{(1+4 \gamma t)^{\frac{d}{2}\left(\frac{1}{p}+\frac{1}{q}\right)}}{(4 \pi t)^{\frac{d}{2}\left(\frac{1}{p}-\frac{1}{q}\right)}}\|\varphi\|_{p,-\gamma} .
$$


Then (6.6) gives

$$
\left(\frac{\pi}{\gamma(t)}\right)^{\frac{d}{2}\left(1-\frac{1}{q}\right)} q^{d / 2 q}\|S(t) \varphi\|_{L_{-q \gamma(t)}^{q}\left(\mathbb{R}^{d}\right)} \leq \frac{(1+4 \gamma t)^{\frac{d}{2}\left(\frac{1}{p}+\frac{1}{q}\right)}}{(4 \pi t)^{\frac{d}{2}\left(\frac{1}{p}-\frac{1}{q}\right)}}\left(\frac{\pi}{\gamma}\right)^{\frac{d}{2}\left(1-\frac{1}{p}\right)} p^{d / 2 p}\|\varphi\|_{L_{-p \gamma(t)}^{p}\left(\mathbb{R}^{d}\right)}
$$

and we get (6.14).

We can summarise some of the estimates above in a compact way if for $\gamma>0$ we define

$$
Y_{-\gamma}^{p}= \begin{cases}L_{-p \gamma}^{p}\left(\mathbb{R}^{d}\right), & \text { if } 1 \leq p<\infty \\ L_{-\gamma}^{p}\left(\mathbb{R}^{d}\right), & \text { if } p=\infty\end{cases}
$$

cf. (4.18).

Corollary 6.8. For any $t>0$ and $1 \leq p \leq q \leq \infty$ (even $t=0$ if $p=q)$

$$
S(t): Y_{-\gamma}^{p}\left(\mathbb{R}^{d}\right) \longrightarrow Y_{-\gamma(t)}^{q}\left(\mathbb{R}^{d}\right), \quad \gamma(t)=\frac{\gamma}{1+4 \gamma t},
$$

is continuous. We can therefore define

$$
S(t): L_{-0}^{p}\left(\mathbb{R}^{d}\right) \rightarrow L_{-0}^{q}\left(\mathbb{R}^{d}\right)
$$

for any $t>0$ and $1 \leq p \leq q \leq \infty$ (even $t=0$ if $p=q$ ), and $S(\cdot)$ satisfies the semigroup property if $p=q$,

$$
S(t+s)=S(t) \circ S(s), \quad t, s \geq 0 .
$$

We can obtain the following estimates on derivatives.

Proposition 6.9. For any multi-index $\alpha \in \mathbb{N}^{d}$ the following estimates hold

$$
\begin{gathered}
\left\|D_{x}^{\alpha} S(t) \varphi\right\|_{L_{-\tilde{\gamma}(t)}^{\infty}\left(\mathbb{R}^{d}\right)} \leq \frac{c}{t^{|\alpha| / 2}}\|\varphi\|_{L_{-\gamma}^{\infty}\left(\mathbb{R}^{d}\right)}, \\
\left\|D_{x}^{\alpha} S(t) \varphi\right\|_{L_{-\tilde{\gamma}(t)}^{\infty}\left(\mathbb{R}^{d}\right)} \leq \frac{c}{t^{|\alpha| / 2}}\left(\frac{1+4 \gamma \delta t}{t}\right)^{d / 2}\|\varphi\|_{\mathcal{M}_{-\gamma}\left(\mathbb{R}^{d}\right)},
\end{gathered}
$$

and

$$
\left\|D_{x}^{\alpha} S(t) \varphi\right\|_{L_{-\tilde{\gamma}(t)}^{1}\left(\mathbb{R}^{d}\right)} \leq \frac{c}{t^{|\alpha| / 2}}(1+4 \gamma \delta t)^{d}\|\varphi\|_{\mathcal{M}_{-\gamma}\left(\mathbb{R}^{d}\right)},
$$

wher $\tilde{\gamma}(t)=\frac{\gamma}{1+4 \gamma \delta t}$ and $\delta>1$.

Hence

(i) for $1 \leq p<\infty$ and $\varphi \in \mathcal{M}_{-\gamma}\left(\mathbb{R}^{d}\right)$

$$
\left\|D_{x}^{\alpha} S(t) \varphi\right\|_{L_{-p \tilde{\gamma}(t)}^{p}\left(\mathbb{R}^{d}\right)} \leq \frac{c}{t^{|\alpha| / 2}} \frac{(1+4 \gamma \delta t)^{\frac{d}{p}}}{t^{\frac{d}{2}\left(1-\frac{1}{p}\right)}}\|\varphi\|_{\mathcal{M}_{-\gamma}\left(\mathbb{R}^{d}\right)} .
$$


(ii) For $1 \leq p<\infty$ and $\varphi \in L_{-p \gamma}^{p}\left(\mathbb{R}^{d}\right)$

$$
\left\|D_{x}^{\alpha} S(t) \varphi\right\|_{L_{-\tilde{\gamma}(t)}^{\infty}\left(\mathbb{R}^{d}\right)} \leq \frac{c}{t^{|\alpha| / 2}}\left(\frac{1+4 \gamma \delta t}{t}\right)^{\frac{d}{2 p}}\|\varphi\|_{L_{-p \gamma}^{p}\left(\mathbb{R}^{d}\right)} .
$$

(iii) For $1 \leq p \leq q<\infty$ and $\varphi \in L_{-p \gamma}^{p}\left(\mathbb{R}^{d}\right)$

$$
\left\|D_{x}^{\alpha} S(t) \varphi\right\|_{L_{-q \tilde{\gamma}(t)}^{q}\left(\mathbb{R}^{d}\right)} \leq \frac{c}{t^{|\alpha| / 2}} \frac{(1+4 \gamma \delta t)^{\frac{d}{2}\left(\frac{1}{p}+\frac{2}{q}-1\right)}}{t^{\frac{d}{2}\left(\frac{1}{p}-\frac{1}{q}\right)}}\|\varphi\|_{L_{-p \gamma}^{p}\left(\mathbb{R}^{d}\right)} .
$$

Proof. For $\varphi \in \mathcal{M}_{-\gamma}\left(\mathbb{R}^{d}\right)$ and any multi-index $\alpha \in \mathbb{N}^{d}$ and any $0<\beta<1$

$$
\left|D_{x}^{\alpha} u(x, t)\right| \leq \frac{c_{\alpha, \beta}}{t^{d / 2+|\alpha| / 2}} \int_{\mathbb{R}^{d}} \mathrm{e}^{-(1-\beta) \frac{|x-y|^{2}}{4 t}} \mathrm{e}^{-\gamma|y|^{2}} \mathrm{e}^{\gamma|y|^{2}} \mathrm{~d}|\varphi(y)|
$$

which is like (6.10) with an extra factor $\frac{1}{t^{|\alpha| / 2}}$ and $t^{\prime}=\delta t$, with $\delta=\frac{1}{1-\beta}>1$, replacing $t$ in the integral term. Therefore we can proceed as in the proof of Proposition 6.6 to obtain (6.20), (6.21), (6.22).

Then interpolating as in Corollary 6.7 we get (6.23), (6.24), (6.25).

\subsection{Dual heat flows}

Now our goal is to show that the heat flow in the spaces $L_{\varepsilon}^{p}\left(\mathbb{R}^{d}\right)$ is the adjoint of the heat flow in the spaces $L_{-\gamma}^{p}\left(\mathbb{R}^{d}\right)$. A first indication for this comes from Theorem 2.1 from where we have that for every $u_{0} \in \mathcal{M}_{\varepsilon}\left(\mathbb{R}^{d}\right)$ and $\varphi \in C_{c}\left(\mathbb{R}^{d}\right)$ and $0 \leq t<T(\varepsilon)=\frac{1}{4 \varepsilon}$

$$
\int_{\mathbb{R}^{d}} \varphi S(t) u_{0}=\int_{\mathbb{R}^{d}} S(t) \varphi \mathrm{d} u_{0}
$$

To prove this we will need the following preparatory result.

Lemma 6.10. Assume that $\mu \in \mathcal{M}_{\varepsilon}\left(\mathbb{R}^{d}\right)$ and $\phi \in \mathcal{M}_{-\gamma}\left(\mathbb{R}^{d}\right)$ with $\gamma>\varepsilon$, define $T(\varepsilon)=\frac{1}{4 \varepsilon}$, $T(\gamma)=\frac{1}{4 \gamma}$, and set $K(x, t)=(4 \pi t)^{-d / 2} \mathrm{e}^{-\frac{|x|^{2}}{4 t}}$.

Then for every $0<t \leq T(\varepsilon)-T(\gamma)=\frac{1}{4 \varepsilon}-\frac{1}{4 \gamma}$

$$
\int_{\mathbb{R}^{d}} \int_{\mathbb{R}^{d}} K(x-y, t) \mathrm{d}|\phi(x)| \mathrm{d}|\mu(y)| \leq \frac{1}{(4 \pi t(1-4 \varepsilon t))^{d / 2}}\|\mu\|_{\mathcal{M}_{\varepsilon}\left(\mathbb{R}^{d}\right)}\|\phi\|_{\mathcal{M}_{-\varepsilon(t)}\left(\mathbb{R}^{d}\right)}
$$

with $\varepsilon(t)=\frac{1}{4(T(\varepsilon)-t)}=\frac{\varepsilon}{1-4 \varepsilon t}$ and

$$
\int_{\mathbb{R}^{d}} \int_{\mathbb{R}^{d}} K(x-y, t) \mathrm{d}|\phi(x)| \mathrm{d}|\mu(y)| \leq\left(\frac{1+4 \gamma t}{4 \pi t}\right)^{d / 2}\|\mu\|_{\mathcal{M}_{\gamma(t)}\left(\mathbb{R}^{d}\right)}\|\phi\|_{\mathcal{M}_{-\gamma}\left(\mathbb{R}^{d}\right)}
$$

with $\gamma(t)=\frac{\gamma}{1+4 \gamma t}$. Moreover, the inequalities above become equalities if $\mu, \phi \geq 0$.

In particular, for $0<t \leq T(\varepsilon)-T(\gamma)=\frac{1}{4 \varepsilon}-\frac{1}{4 \gamma}$

$$
\int_{\mathbb{R}^{d}} S(t) \mu \mathrm{d} \phi=\int_{\mathbb{R}^{d}} S(t) \phi \mathrm{d} \mu .
$$


Proof. (i) Notice that

$$
I=\int_{\mathbb{R}^{d}} \int_{\mathbb{R}^{d}} K(x-y, t) \mathrm{d}|\phi(x)| \mathrm{d}|\mu(y)|=\int_{\mathbb{R}^{d}} \int_{\mathbb{R}^{d}} K(x-y, t) \mathrm{e}^{\varepsilon|y|^{2}} \mathrm{e}^{-\varepsilon|y|^{2}} \mathrm{~d}|\phi(x)| \mathrm{d}|\mu(y)|
$$

and completing the square

$$
\frac{|x-y|^{2}}{4 t}-\varepsilon|y|^{2}=\frac{1-4 \varepsilon t}{4 t}\left|y-\frac{1}{1-4 \varepsilon t} x\right|^{2}-\frac{\varepsilon|x|^{2}}{1-4 \varepsilon t} .
$$

Hence

$$
\begin{aligned}
I & \leq(4 \pi t)^{-d / 2} \int_{\mathbb{R}^{d}} \int_{\mathbb{R}^{d}} \mathrm{e}^{-\frac{1-4 \varepsilon t}{4 t}\left|y-\frac{1}{1-4 \varepsilon t} x\right|^{2}} \mathrm{e}^{\frac{\varepsilon|x|^{2}}{1-4 \varepsilon t}} \mathrm{e}^{-\varepsilon|y|^{2}} \mathrm{~d}|\phi(x)| \mathrm{d}|\mu(y)| \\
& \leq(4 \pi t)^{-d / 2} \int_{\mathbb{R}^{d}} \int_{\mathbb{R}^{d}} \mathrm{e}^{\frac{\varepsilon|x|^{2}}{1-4 \varepsilon t}} \mathrm{e}^{-\varepsilon|y|^{2}} \mathrm{~d}|\phi(x)| \mathrm{d}|\mu(y)| \\
& =(4 \pi t)^{-d / 2} \int_{\mathbb{R}^{d}} \mathrm{e}^{-\varepsilon|y|^{2}} \mathrm{~d}|\mu(y)| \int_{\mathbb{R}^{d}} \mathrm{e}^{\frac{\varepsilon|x|^{2}}{1-4 \varepsilon t}} \mathrm{~d}|\phi(x)| \\
& =(4 \pi t)^{-d / 2}\left(\frac{\pi}{\varepsilon}\right)^{\frac{d}{2}}\left(\frac{\varepsilon(t)}{\pi}\right)^{\frac{d}{2}}\|\mu\|_{\mathcal{M}_{\varepsilon}\left(\mathbb{R}^{d}\right)}\|\phi\|_{\mathcal{M}_{-\varepsilon(t)}\left(\mathbb{R}^{d}\right)} \\
& =(4 \pi t)^{-d / 2}\left(\frac{\varepsilon(t)}{\varepsilon}\right)^{\frac{d}{2}}\|\mu\|_{\mathcal{M}_{\varepsilon}\left(\mathbb{R}^{d}\right)}\|\phi\|_{\mathcal{M}_{-\varepsilon(t)}\left(\mathbb{R}^{d}\right)},
\end{aligned}
$$

which is finite as long as $\varepsilon(t) \leq \gamma$, that is $0<t \leq T(\varepsilon)-T(\gamma)=\frac{1}{4 \varepsilon}-\frac{1}{4 \gamma}$.

(ii) Observe now that

$$
I=\int_{\mathbb{R}^{d}} \int_{\mathbb{R}^{d}} K(x-y, t) \mathrm{d}|\phi(x)| \mathrm{d}|\mu(y)|=\int_{\mathbb{R}^{d}} \int_{\mathbb{R}^{d}} K(x-y, t) \mathrm{e}^{-\gamma|x|^{2}} \mathrm{e}^{\gamma|x|^{2}} \mathrm{~d}|\phi(x)| \mathrm{d}|\mu(y)|
$$

and completing the square

$$
\frac{|x-y|^{2}}{4 t}+\gamma|x|^{2}=\frac{1+4 \gamma t}{4 t}\left|x-\frac{1}{1+4 \gamma t} y\right|^{2}+\frac{\gamma|y|^{2}}{1+4 \gamma t} .
$$

Hence with $\gamma(t)=\frac{\gamma}{1+4 \gamma t}$ we have

$$
\begin{aligned}
I & \leq(4 \pi t)^{-d / 2} \int_{\mathbb{R}^{d}} \int_{\mathbb{R}^{d}} \mathrm{e}^{-\frac{1+4 \gamma t}{4 t}\left|x-\frac{1}{1+4 \gamma t} y\right|^{2}} \mathrm{e}^{\frac{-\gamma|y|^{2}}{1+4 \gamma t}} \mathrm{e}^{\gamma|x|^{2}} \mathrm{~d}|\phi(x)| \mathrm{d}|\mu(y)| \\
& \leq(4 \pi t)^{-d / 2} \int_{\mathbb{R}^{d}} \int_{\mathbb{R}^{d}} \mathrm{e}^{\frac{-\gamma|y|^{2}}{1+4 \gamma t}} \mathrm{e}^{\gamma|x|^{2}} \mathrm{~d}|\phi(x)| \mathrm{d}|\mu(y)| \\
& =(4 \pi t)^{-d / 2} \int_{\mathbb{R}^{d}} \mathrm{e}^{\gamma|x|^{2}} \mathrm{~d}|\phi(x)| \int_{\mathbb{R}^{d}} \mathrm{e}^{\frac{-\gamma|y|^{2}}{1+4 \gamma t}} \mathrm{~d}|\mu(y)| \\
& =(4 \pi t)^{-d / 2}\left(\frac{\pi}{\gamma(t)}\right)^{\frac{d}{2}}\left(\frac{\gamma}{\pi}\right)^{\frac{d}{2}}\|\mu\|_{\mathcal{M}_{\gamma(t)}\left(\mathbb{R}^{d}\right)}\|\phi\|_{\mathcal{M}_{-\gamma}\left(\mathbb{R}^{d}\right)} \\
& =(4 \pi t)^{-d / 2}\left(\frac{\gamma}{\gamma(t)}\right)^{\frac{d}{2}}\|\mu\|_{\mathcal{M}_{\gamma(t)}\left(\mathbb{R}^{d}\right)}\|\phi\|_{\mathcal{M}_{-\gamma}\left(\mathbb{R}^{d}\right)},
\end{aligned}
$$

which is finite as long as $\gamma(t) \geq \varepsilon$, that is $0<t \leq T(\varepsilon)-T(\gamma)=\frac{1}{4 \varepsilon}-\frac{1}{4 \gamma}$. 
Then (6.26) follows from Fubini's theorem since

$$
\begin{aligned}
\int_{\mathbb{R}^{d}} S(t) \mu \mathrm{d} \phi & =\int_{\mathbb{R}^{d}} \int_{\mathbb{R}^{d}} K(x-y, t) \mathrm{d} \mu(x) \mathrm{d} \phi(y) \\
& =\int_{\mathbb{R}^{d}} \int_{\mathbb{R}^{d}} K(x-y, t) \mathrm{d} \phi(y) \mathrm{d} \mu(x) \\
& =\int_{\mathbb{R}^{d}} S(t) \phi \mathrm{d} \mu .
\end{aligned}
$$

Note that Proposition 6.4 establishes a duality among the spaces $X_{\varepsilon}^{p}$ from (4.18) and the spaces $Y_{-\gamma}^{q}$ from (6.19). Also the norms $\|\cdot \cdot\|_{p, \varepsilon}$ and $\|\cdot \cdot\|_{p^{\prime},-\varepsilon}$ are dual to each other. In particular, using Proposition 4.10, we have the following result.

Proposition 6.11. Defining $X_{\varepsilon}^{p}$ and $Y_{-\varepsilon}^{p}$ as in (4.18) and (6.19), for any $\delta>0, t>0$ and $\delta(t)=\frac{\delta}{1+4 \delta t}, 1 \leq p \leq \infty, \frac{1}{p}+\frac{1}{p^{\prime}}=1$ and any $u_{0} \in X_{\delta(t)}^{p}$ and $\varphi \in Y_{-\delta}^{p^{\prime}}$, we have

$$
\int_{\mathbb{R}^{d}} \varphi S(t) u_{0}=\int_{\mathbb{R}^{d}} S(t) \varphi u_{0}
$$

Moreover in all the pairs of estimates (3.2)-(6.7), (4.9)-(6.13), (4.10)-(6.14), (4.6)-(6.9), and (4.11)-(6.12) one estimate is dual of the other.

Proof. With the spaces in (6.5), the definitions in (4.18) and (6.19), from (4.19) we have that

$$
S(t): X_{\delta(t)}^{p} \rightarrow X_{\delta}^{q}
$$

is continuous for $1 \leq p \leq q \leq \infty$.

Hence, for $q<\infty$, using Proposition 6.4, the adjoint operator satisfies

$$
S^{\prime}(t): Y_{-\delta}^{q^{\prime}} \rightarrow Y_{-\delta(t)}^{p^{\prime}}
$$

for $1<q^{\prime} \leq p^{\prime} \leq \infty$ and is characterized by

$$
\int_{\mathbb{R}^{d}} S(t) u_{0} \varphi=\int_{\mathbb{R}^{d}} u_{0} S^{\prime}(t) \varphi
$$

for any $u_{0} \in X_{\delta(t)}^{p}$ and $\varphi \in Y_{-\delta}^{q^{\prime}}$. Hence, from Lemma 6.10 we get $S^{\prime}(t)=S(t)$.

Finally, since from Proposition 6.4 the norms $\|\cdot \mid\|_{p, \varepsilon}$ and $\|\cdot \mid\|_{p^{\prime},-\varepsilon}$ are dual to each other, the adjoint operator satisfies the dual estimates (6.7), (6.13) (from (6.16)), and (6.14) (from (6.18)), respectively.

Conversely, the estimates (6.9) in Proposition 6.6 and (6.14) (from (6.18)), (6.12) (from (6.15)) in Corollary 6.7 imply that

$$
S(t): Y_{-\delta}^{p} \rightarrow Y_{-\delta(t)}^{q}
$$


is continuous for $1 \leq p \leq q<\infty$. Hence the adjoint operator satisfies

$$
S^{\prime}(t): X_{\delta(t)}^{q^{\prime}} \rightarrow X_{\delta}^{p^{\prime}}
$$

for $1<q^{\prime} \leq p^{\prime} \leq \infty$. As above, from Lemma 6.10, $S^{\prime}(t)=S(t)$ and again from Proposition 6.4 the adjoint satisfies the dual estimates (4.6), (4.10) and (4.11) respectively.

As in Proposition 3.4 and Proposition 4.11, we can recast the results in Proposition 6.11 as follows.

Corollary 6.12. For any $\varepsilon>0,0<t<T(\varepsilon)=\frac{1}{4 \varepsilon}$, we have the following.

For any $1 \leq p \leq \infty, \frac{1}{p}+\frac{1}{p^{\prime}}=1$ and any $u_{0} \in X_{\varepsilon}^{p}$ and $\varphi \in Y_{-\varepsilon(t)}^{p^{\prime}}$, we have

$$
\int_{\mathbb{R}^{d}} \varphi S(t) u_{0}=\int_{\mathbb{R}^{d}} S(t) \varphi u_{0}
$$

Proof. As in the Proposition above for $\delta>0, t>0$ and $\delta(t)=\frac{\delta}{1+4 \delta t}<\delta$, we have (4.19) for $1 \leq p \leq q \leq \infty$.

So given $\varepsilon>0$ and $0<t<T(\varepsilon)$ set $\delta=\varepsilon(t)=\frac{1}{4(T(\varepsilon)-t)}=\frac{\varepsilon}{(1-4 \varepsilon t)}>\varepsilon$ and then $\delta(t)=\varepsilon$. This proves (i).

Also (ii) follows in the same way from (6.27).

Finally in the Fréchet space $L_{0}^{p}\left(\mathbb{R}^{d}\right)$ we have the following.

Proposition 6.13. For $1 \leq p \leq q<\infty$, the heat flow $S(t): L_{-0}^{q^{\prime}}\left(\mathbb{R}^{d}\right) \rightarrow L_{-0}^{p^{\prime}}\left(\mathbb{R}^{d}\right)$ as in Corollary 6.8 is the adjoint of the heat flow $S(t): L_{0}^{p}\left(\mathbb{R}^{d}\right) \rightarrow L_{0}^{q}\left(\mathbb{R}^{d}\right)$ as in Proposition 4.8 .

In particular, for all $u_{0} \in L_{0}^{p}\left(\mathbb{R}^{d}\right), v_{0} \in L_{-0}^{p^{\prime}}\left(\mathbb{R}^{d}\right)$ and $t>0$, we have

$$
\int_{\mathbb{R}^{d}} v_{0} S(t) u_{0}=\int_{\mathbb{R}^{d}} S(t) v_{0} u_{0}
$$

Proof. For $1 \leq p \leq q<\infty$ from Proposition 4.8, $S(t): L_{0}^{p}\left(\mathbb{R}^{d}\right) \rightarrow L_{0}^{q}\left(\mathbb{R}^{d}\right)$ is continuous. Hence the adjoint satisfies $S^{\prime}(t):\left(L_{0}^{q}\left(\mathbb{R}^{d}\right)\right)^{\prime} \rightarrow\left(L_{0}^{p}\left(\mathbb{R}^{d}\right)\right)^{\prime}$ and is definded by

$$
\int_{\mathbb{R}^{d}} S(t) u_{0} \varphi=\int_{\mathbb{R}^{d}} u_{0} S^{\prime}(t) \varphi
$$

for $u_{0} \in L_{0}^{p}\left(\mathbb{R}^{d}\right)$ and $\varphi \in\left(L_{0}^{q}\left(\mathbb{R}^{d}\right)\right)^{\prime}$. By Corollary 6.5 and Lemma 6.10 we conclude that $S^{\prime}(t) \varphi=S(t) \varphi$. 


\section{Conclusion}

The results in this paper show that the heat equation defines, in a very natural way, a semigroup on the family of Fréchet spaces $L_{0}^{p}\left(\mathbb{R}^{d}\right)$, and that we need to use the whole family of seminorms of $L_{0}^{p}\left(\mathbb{R}^{d}\right)$ in order to understand the behaviour of the heat flow for such initial data.

We have also developed a systematic dual theory for both the $L_{0}^{p}\left(\mathbb{R}^{d}\right)$ spaces and the heat flow on these spaces.

We will use the estimates from the first part of the paper to analyse the inhomogeneous equation

$$
u_{t}-\Delta u+\lambda u=f(t)
$$

and deduce consequences for the elliptic equation

$$
-\Delta u+\lambda u=f
$$

for $f \in L_{0}^{p}\left(\mathbb{R}^{d}\right)$ in our forthcoming paper [7].

\section{References}

[1] J. Bergh, J. Löfström, Interpolation spaces. An introduction, Springer-Verlag 1976.

[2] G.B. Folland, Real analysis. Modern techniques and their applications, Wiley 1990.

[3] P. Fitzpatrick, H. Royden, Real Analysis (4th Edition), Prentice Hall, 2010

[4] A. Lunardi, "Analytic semigroups and optimal regularity in parabolic problems". Basel: Birkhuser Verlag, 1995

[5] A. Pazy, "Semigroups of Linear Operators and Applications to Partial Differential Equations". Springer-Verlag New York, Inc., 1983.

[6] J.C. Robinson, A. Rodriguez-Bernal, Optimal existence classes and nonlinear-like dynamics in the linear heat equation in $\mathbb{R}^{d}$, Advances in Mathematics 334, 488-543 (2018).

[7] J.C. Robinson, A. Rodriguez-Bernal, Linear non-autonomous heat flow in $L_{0}^{1}\left(\mathbb{R}^{d}\right)$ and applications to elliptic equations in $\mathbb{R}^{d}$. In preparation. 